\title{
WEIGHTED VECTOR-VALUED INEQUALITIES FOR A \\ CLASS OF MULTILINEAR SINGULAR INTEGRAL OPERATORS
}

\section{$\mathrm{Hu}$, Guoen}

2018-05

Hu , G \& Li , K 2018 , ' WEIGHTED VECTOR-VALUED INEQUALITIES FOR A CLASS OF MULTILINEAR SINGULAR INTEGRAL OPERATORS ' , Proceedings of the Edinburgh Mathematical Society , vol. 61 , no. 2 , pp. 413-436 . https://doi.org/10.1017/S0013091517000153

http://hdl.handle.net/10138/307583

https://doi.org/10.1017/S0013091517000153

cc_by_nc_nd

acceptedVersion

Downloaded from Helda, University of Helsinki institutional repository.

This is an electronic reprint of the original article.

This reprint may differ from the original in pagination and typographic detail.

Please cite the original version. 


\title{
WEIGHTED VECTOR-VALUED INEQUALITIES FOR A CLASS OF MULTILINEAR SINGULAR INTEGRAL OPERATORS
}

\author{
GUOEN HU AND KANGWEI LI
}

\begin{abstract}
In this paper, some weighted vector-valued inequalities with multiple weights $A_{\vec{P}}\left(\mathbb{R}^{m n}\right)$ are established for a class of multilinear singular integral operators. The weighted estimates for the multi(sub)linear maximal operators which control the multilinear singular integral operators are also considered.
\end{abstract}

\section{INTRODUCTION}

In recent years, considerable attention has been paid to the boundedness of the multilinear singular integral operator on function spaces. Let $K\left(x ; y_{1}, \ldots, y_{m}\right)$ be a locally integrable function defined away from the diagonal $x=y_{1}=\cdots=y_{m}$ in $\mathbb{R}^{m n}$. An operator $T$ defined on $\mathcal{S}\left(\mathbb{R}^{n}\right) \times \cdots \times \mathcal{S}\left(\mathbb{R}^{n}\right)$ (Schwartz space) and taking values in $\mathcal{S}^{\prime}\left(\mathbb{R}^{n}\right)$, is said to be an $m$-multilinear singular integral operator with kernel $K$, if $T$ is $m$-multilinear, and satisfies that

$$
T\left(f_{1}, \ldots, f_{m}\right)(x)=\int_{\mathbb{R}^{m n}} K\left(x ; y_{1}, \ldots, y_{m}\right) \prod_{j=1}^{m} f_{j}\left(y_{j}\right) \mathrm{d} \vec{y},
$$

for bounded functions $f_{1}, \ldots, f_{m}$ with compact supports, and $x \in \mathbb{R}^{n} \backslash \cap_{j=1}^{m} \operatorname{supp} f_{j}$. Operators of this type were originated in the remarkable works of Coifman and Meyer [2], [3], and are useful in multilinear analysis. We say that $K$ is a multilinear Calderón-Zygmund kernel, if $K$ satisfies the size condition that for all $\left(x, y_{1}, \ldots, y_{m}\right) \in \mathbb{R}^{(m+1) n}$ with $x \neq y_{j}$ for some $1 \leq j \leq m$,

$$
\left|K\left(x ; y_{1}, \ldots, y_{m}\right)\right| \lesssim \frac{1}{\left(\sum_{j=1}^{m}\left|x-y_{j}\right|\right)^{m n}}
$$

and satisfies the regularity condition that for some $\beta \in(0,1]$

$$
\left|K\left(x ; y_{1}, \ldots, y_{m}\right)-K\left(x^{\prime} ; y_{1}, \ldots, y_{m}\right)\right| \lesssim \frac{\left|x-x^{\prime}\right|^{\beta}}{\left(\sum_{j=1}^{m}\left|x-y_{j}\right|\right)^{m n+\beta}}
$$

whenever $\max _{1 \leq k \leq m}\left|x-y_{k}\right| \geq 2\left|x-x^{\prime}\right|$, and for all $1 \leq j \leq m$,

$$
\left|K\left(x ; y_{1}, \ldots, y_{j} \ldots, y_{m}\right)-K\left(x ; y_{1}, \ldots, y_{j}^{\prime}, \ldots, y_{m}\right)\right| \lesssim \frac{\left|y_{j}-y_{j}^{\prime}\right|^{\beta}}{\left(\sum_{i=1}^{m}\left|x-y_{i}\right|\right)^{m n+\beta}}
$$

whenever $\max _{1 \leq k \leq m}\left|x-y_{k}\right| \geq 2\left|y_{j}-y_{j}^{\prime}\right|$. When $K$ is a multilinear CalderónZygmund kernel, Grafakos and Torres [12] considered the behavior of $T$ on $L^{1}\left(\mathbb{R}^{n}\right) \times$ $\cdots \times L^{1}\left(\mathbb{R}^{n}\right)$, and established a $T 1$ type theorem for the operator $T$. Lerner, Ombrossi, Pérez, Torres and Trojillo-Gonzalez [16] introduced a new maximal operator and a new class of multiple weights $A_{\vec{P}}\left(\mathbb{R}^{m n}\right)$ (see Definition 1.9 below), and established the weighted estimates with $A_{\vec{P}}\left(\mathbb{R}^{m n}\right)$ for the multilinear Calderón-Zygmund

2010 Mathematics Subject Classification. 42B20.

Key words and phrases. Vector-valued inequality, multilinear singular integral operator, nonsmooth kernel, multiple weight. 
singular integral operators. For other mapping properties of multilinear CalderónZygmund operators on various function spaces, see $[9,11,12,17,18]$ and references therein.

To study the mapping properties for the commutator of Calderón, Duong, Grafakos and Yan [6] introduced a class of multilinear singular integral operators via the following generalized approximation to the identity.

Definition 1.3. A family of operators $\left\{A_{t}\right\}_{t>0}$ is said to be an approximation to the identity, if for every $t>0, A_{t}$ can be represented by the kernel at in the following sense: for every function $u \in L^{p}\left(\mathbb{R}^{n}\right)$ with $p \in[1, \infty]$ and almost everywhere $x \in \mathbb{R}^{n}$,

$$
A_{t} u(x)=\int_{\mathbb{R}^{n}} a_{t}(x, y) u(y) \mathrm{d} y,
$$

and the kernel $a_{t}$ satisfies that for all $x, y \in \mathbb{R}^{n}$ and $t>0$,

$$
\left|a_{t}(x, y)\right| \leq h_{t}(x, y)=t^{-n / s} h\left(\frac{|x-y|}{t^{1 / s}}\right),
$$

where $s>0$ is a constant and $h$ is a positive, bounded and decreasing function such that for some constant $\eta>0$,

$$
\lim _{r \rightarrow \infty} r^{n+\eta} h\left(r^{s}\right)=0 .
$$

Assumption 1.6. For each fixed $j$ with $1 \leq j \leq m$, there exists an approximation to the identity $\left\{A_{t}^{j}\right\}_{t>0}$ with kernels $\left\{a_{t}^{j}(x, y)\right\}_{t>0}$, and there exist kernels $K_{t}^{j}\left(x ; y_{1}, \ldots, y_{m}\right)$, such that for bounded functions $f_{1}, \ldots, f_{m}$ with compact supports, and $x \in \mathbb{R}^{n} \backslash \cap_{k=1}^{m} \operatorname{supp} f_{k}$,

$$
T\left(f_{1}, \ldots, f_{j-1}, A_{t}^{j} f_{j}, f_{j+1} \ldots, f_{m}\right)(x)=\int_{\mathbb{R}^{n m}} K_{t}^{j}\left(x ; y_{1}, \ldots, y_{m}\right) \prod_{k=1}^{m} f_{k}\left(y_{k}\right) \mathrm{d} \vec{y},
$$

and there exists a function $\phi \in C(\mathbb{R})$ with $\operatorname{supp} \phi \subset[-1,1]$, and a constant $\varepsilon \in$ $(0,1]$, such that for all $x, y_{1}, \ldots, y_{m} \in \mathbb{R}^{n}$ and all $t>0$ with $2 t^{1 / s} \leq\left|x-y_{j}\right|$,

$$
\begin{aligned}
& \left|K\left(x ; y_{1}, \ldots, y_{m}\right)-K_{t}^{j}\left(x ; y_{1}, \ldots, y_{m}\right)\right| \\
& \quad \lesssim \frac{t^{\varepsilon / s}}{\left(\sum_{k=1}^{m}\left|x-y_{k}\right|\right)^{m n+\varepsilon}}+\frac{1}{\left(\sum_{k=1}^{m}\left|x-y_{k}\right|\right)^{m n}} \sum_{1 \leq i \leq m, i \neq j} \phi\left(\frac{\left|y_{i}-y_{j}\right|}{t^{1 / s}}\right) .
\end{aligned}
$$

As it was pointed out in [6], operators with such kernels are called multilinear singular integral operators with non-smooth kernels, since the kernel $K$ satisfying Assumption 1.6 may enjoy no smoothness in the variables $y_{1} \ldots, y_{m}$. Duong, Grafakos and Yan proved that if $T$ satisfies Assumption 1.6, and is bounded from $L^{q_{1}}\left(\mathbb{R}^{n}\right) \times \cdots \times L^{q_{m}}\left(\mathbb{R}^{n}\right)$ to $L^{q, \infty}\left(\mathbb{R}^{n}\right)$ for some $q_{1}, \ldots, q_{m} \in(1, \infty)$ and $q \in(0, \infty)$ with $1 / q=1 / q_{1}+\cdots+1 / q_{m}$, then $T$ is also bounded from $L^{1}\left(\mathbb{R}^{n}\right) \times \cdots \times L^{1}\left(\mathbb{R}^{n}\right)$ to $L^{1 / m, \infty}\left(\mathbb{R}^{n}\right)$. Let $T^{*}$ be the maximal operator associated with the operator $T$ satisfing Assumption 1.6, that is,

$$
T^{*}\left(f_{1}, \ldots, f_{m}\right)(x)=\sup _{\epsilon>0}\left|\int_{\sum_{j=1}^{m}|x-y|^{2}>\epsilon^{2}} K\left(x ; y_{1}, \ldots, y_{m}\right) \prod_{j=1}^{m} f_{j}\left(y_{j}\right) \mathrm{d} \vec{y}\right| .
$$

To consider the weighted estimates with $A_{p}\left(\mathbb{R}^{n}\right)$ weights for $T^{*}$, Duong et al. [5] introduced the following two assumptions.

Assumption 1.7. There exists an approximation to the identity $\left\{B_{t}\right\}_{t>0}$ with kernels $\left\{b_{t}(x, y)\right\}_{t>0}$, and there exist kernels $\left\{K_{t}^{0}\left(x ; y_{1}, \ldots, y_{m}\right)\right\}_{t>0}$ such that

$$
K_{t}^{0}\left(x ; y_{1}, \ldots, y_{m}\right)=\int_{\mathbb{R}^{n}} K\left(z ; y_{1}, \ldots, y_{m}\right) b_{t}(x, z) \mathrm{d} z
$$


and there exists a function $\psi \in C(\mathbb{R})$ with $\operatorname{supp} \psi \subset[-1,1]$, and a constant $\gamma \in(0,1]$, such that for all $x, y_{1}, \ldots, y_{m} \in \mathbb{R}^{n}$ and all $t>0$ with $2 t^{1 / s} \leq$ $\max _{1 \leq k \leq m}\left|x-y_{k}\right|$,

$$
\begin{aligned}
& \left|K\left(x ; y_{1}, \ldots, y_{m}\right)-K_{t}^{0}\left(x ; y_{1}, \ldots, y_{m}\right)\right| \\
& \quad \lesssim \frac{t^{\gamma / s}}{\left(\sum_{k=1}^{m}\left|x-y_{k}\right|\right)^{m n+\gamma}}+\frac{1}{\left(\sum_{k=1}^{m}\left|x-y_{k}\right|\right)^{m n}} \sum_{1 \leq j \leq m} \psi\left(\frac{\left|x-y_{j}\right|}{t^{1 / s}}\right) .
\end{aligned}
$$

Assumption 1.8. The kernel $K_{t}^{0}\left(x ; y_{1}, \ldots, y_{m}\right)$ in Assumption 1.7 satisfies the size condition that

$$
\left|K_{t}^{0}\left(x ; y_{1}, \ldots, y_{m}\right)\right| \lesssim \frac{1}{\left(\sum_{j=1}^{m}\left|x-y_{j}\right|\right)^{m n}}
$$

whenever $2 t^{1 / s} \leq \min _{1 \leq j \leq m}\left|x-y_{j}\right|$, and the regularity condition that

$$
\left|K_{t}^{0}\left(x ; y_{1}, \ldots, y_{m}\right)-K_{t}^{0}\left(x^{\prime} ; y_{1}, \ldots, y_{m}\right)\right| \lesssim \frac{t^{\gamma / s}}{\left(\sum_{j=1}^{m}\left|x-y_{j}\right|\right)^{m n+\gamma}}
$$

whenever $2\left|x-x^{\prime}\right| \leq t^{1 / s}$ and $2 t^{1 / s} \leq \min _{1 \leq j \leq m}\left|x-y_{j}\right|$.

Duong et al. [5] proved that if $T$ satisfies Assumption 1.6, Assumption 1.7 and Assumption 1.8, and is bounded from $L^{q_{1}}\left(\mathbb{R}^{n}\right) \times \cdots \times L^{q_{m}}\left(\mathbb{R}^{n}\right)$ to $L^{q, \infty}\left(\mathbb{R}^{n}\right)$ for some $q_{1}, \ldots, q_{m} \in(1, \infty)$ and $q \in(0, \infty)$ with $1 / q=1 / q_{1}+\cdots+1 / q_{m}$, then for $p_{1}, \ldots, p_{m} \in[1, \infty)$ and $p \in(0, \infty)$ with $1 / p=1 / p_{1}+\cdots+1 / p_{m}$, and $w \in A_{\min _{1<j<m} p_{j}}\left(\mathbb{R}^{n}\right)$, both $T$ and $T^{*}$ are bounded from $L^{p_{1}}\left(\mathbb{R}^{n}, w\right) \times \cdots \times$ $L^{p_{m}}\left(\mathbb{R}^{n}, w\right)$ to $L^{p, \infty}\left(\mathbb{R}^{n}, w\right)$, and when $\min _{1 \leq j \leq m} p_{j}>1, T$ and $T^{*}$ are bounded from $L^{p_{1}}\left(\mathbb{R}^{n}, w\right) \times \cdots \times L^{p_{m}}\left(\mathbb{R}^{n}, w\right)$ to $L^{p}\left(\mathbb{R}^{n}, w\right)$. Grafakos, Liu and Yang [10] considered the weighted norm inequalities with multiple weights for $T^{*}$, and proved that $T$ and $T^{*}$ enjoy the weighted estimates with $A_{\vec{P}}\left(\mathbb{R}^{m n}\right)$ weights the same as the multilinear Calderón-Zygmund operators.

The purpose of this paper is to establish some weighted vector-valued inequalities with multiple weights for a class of multilinear singular integral operators, as analogies of the weighted vector-valued inequalities with $A_{p}\left(\mathbb{R}^{n}\right)$ weights for the classical Calderón-Zygmund operators (see [1]) in the setting of multilinear singular integral operators. We remark that the operators we consider here, contain the multilinear Calderón-Zygmund operators and the multilinear singular integral operators with non-smooth kernels as examples, see Remark 1.12 below. To state our results, we first recall some definitions and notations.

Let $p, r \in(0, \infty]$ and $w$ be a weight. As usual, for a sequence of numbers $\left\{a_{k}\right\}_{k=1}^{\infty}$, we denote $\left\|\left\{a_{k}\right\}\right\|_{l^{r}}=\left(\sum_{k}\left|a_{k}\right|^{r}\right)^{1 / r}$. The space $L^{p}\left(l^{r} ; \mathbb{R}^{n}, w\right)$ is defined as

where

$$
L^{p}\left(l^{r} ; \mathbb{R}^{n}, w\right)=\left\{\left\{f_{k}\right\}_{k=1}^{\infty}:\left\|\left\{f_{k}\right\}\right\|_{L^{p}\left(l^{r} ; \mathbb{R}^{n}, w\right)}<\infty\right\}
$$

$$
\left\|\left\{f_{k}\right\}\right\|_{L^{p}\left(l^{r} ; \mathbb{R}^{n}, w\right)}=\left(\int_{\mathbb{R}^{n}}\left\|\left\{f_{k}(x)\right\}\right\|_{l^{r}}^{p} w(x) \mathrm{d} x\right)^{1 / p} .
$$

The space $L^{p, \infty}\left(l^{r} ; \mathbb{R}^{n}, w\right)$ is defined as

$$
L^{p, \infty}\left(l^{r} ; \mathbb{R}^{n}, w\right)=\left\{\left\{f_{k}\right\}_{k=1}^{\infty}:\left\|\left\{f_{k}\right\}\right\|_{L^{p, \infty}\left(l^{r} ; \mathbb{R}^{n}, w\right)}<\infty\right\}
$$

with

$$
\left\|\left\{f_{k}\right\}\right\|_{L^{p, \infty}\left(l^{r} ; \mathbb{R}^{n}, w\right)}^{p}=\sup _{\lambda>0} \lambda^{p} w\left(\left\{x \in \mathbb{R}^{n}:\left\|\left\{f_{k}(x)\right\}\right\|_{l^{r}}>\lambda\right\}\right) .
$$

When $w \equiv 1$, we denote $\left\|\left\{f_{k}\right\}\right\|_{L^{p}\left(l^{r} ; \mathbb{R}^{n}, w\right)}\left(\left\|\left\{f_{k}\right\}\right\|_{L^{p, \infty}\left(l^{r} ; \mathbb{R}^{n}, w\right)}\right)$ by $\left\|\left\{f_{k}\right\}\right\|_{L^{p}\left(l^{r} ; \mathbb{R}^{n}\right)}$ $\left(\left\|\left\{f_{k}\right\}\right\|_{L^{p, \infty}\left(l^{r} ; \mathbb{R}^{n}\right)}\right)$ for simplicity.

The following definition of multiple weights was introduced in [16]. 
Definition 1.9. Let $m \in \mathbb{N}, w_{1}, \ldots, w_{m}$ be weights, $p_{1}, \ldots, p_{m} \in[1, \infty), p \in$ $(0, \infty)$ with $1 / p=1 / p_{1}+\cdots+1 / p_{m}$. Set $\vec{w}=\left(w_{1}, \ldots, w_{m}\right), \vec{P}=\left(p_{1}, \ldots, p_{m}\right)$ and $\nu_{\vec{w}}=\prod_{k=1}^{m} w_{k}^{p / p_{k}}$. We say that $\vec{w} \in A_{\vec{P}}\left(\mathbb{R}^{m n}\right)$ if

$$
\sup _{Q \subset \mathbb{R}^{n}}\left(\frac{1}{|Q|} \int_{Q} \nu_{\vec{w}}(x) \mathrm{d} x\right)^{1 / p} \prod_{k=1}^{m}\left(\frac{1}{|Q|} \int_{Q} w_{k}^{-\frac{1}{p_{k}-1}}(x) \mathrm{d} x\right)^{1-1 / p_{k}}<\infty
$$

when $p_{k}=1,\left(\frac{1}{|Q|} \int_{Q} w_{k}^{-\frac{1}{p_{k}-1}}(x) \mathrm{d} x\right)^{1-1 / p_{k}}$ is understood as $\left(\inf _{Q} w_{k}\right)^{-1}$.

Our first result can be stated as follows.

Theorem 1.10. Let $m \geq 2, T$ be an $m$-linear operator with kernel $K$ in the sense of $(1.1), r_{1}, \ldots r_{m} \in(1, \infty), r \in(0, \infty)$ such that $1 / r=1 / r_{1}+\cdots+1 / r_{m}$. Suppose that

(i) $T$ is bounded from $L^{r_{1}}\left(\mathbb{R}^{n}\right) \times \cdots \times L^{r_{m}}\left(\mathbb{R}^{n}\right)$ to $L^{r}\left(\mathbb{R}^{n}\right)$;

(ii) for $x, x^{\prime}, y_{1}, \ldots, y_{m} \in \mathbb{R}^{n}$ with $8\left|x-x^{\prime}\right|<\min _{1 \leq j \leq m}\left|x-y_{j}\right|$, and each number $D$ such that $2\left|x-x^{\prime}\right|<D$ and $4 D<\min _{1 \leq j \leq m}\left|x-y_{j}\right|$

$$
\left|K\left(x ; y_{1}, \ldots, y_{m}\right)-K\left(x^{\prime} ; y_{1}, \ldots, y_{m}\right)\right| \lesssim \frac{D^{\gamma}}{\left(\sum_{j=1}^{m}\left|x-y_{j}\right|\right)^{n m+\gamma}} ;
$$

(iii) T satisfies the size conditon (1.2) and Assumption 1.2.

Let $p_{1}, \ldots, p_{m}, q_{1}, \ldots, q_{m} \in[1, \infty), p, q \in(0,, \infty)$ such that $1 / p=1 / p_{1}+\cdots+$ $1 / p_{m}, 1 / q=1 / q_{1}+\cdots+1 / q_{m}, \vec{w}=\left(w_{1}, \ldots, w_{m}\right) \in A_{\vec{P}}\left(\mathbb{R}^{m n}\right)$. Then $T$ is bounded from $L^{p_{1}}\left(l^{q_{1}} ; \mathbb{R}^{n}, w_{1}\right) \times \cdots \times L^{p_{m}}\left(l^{q_{m}} ; \mathbb{R}^{n}, w_{m}\right)$ to $L^{p, \infty}\left(l^{q} ; \mathbb{R}^{n}, \nu_{\vec{w}}\right)$. Moreover, if $\min _{1 \leq j \leq m} p_{j}>1$, then $T$ is bounded from $L^{p_{1}}\left(l^{q_{1}} ; \mathbb{R}^{n}, w_{1}\right) \times \cdots \times L^{p_{m}}\left(l^{q_{m}} ; \mathbb{R}^{n}, w_{m}\right)$ to $L^{p}\left(l^{q} ; \mathbb{R}^{n}, \nu_{\vec{w}}\right)$.

Remark 1.12. As it was pointed out in [6], if $T$ is an $m$-linear Calderón-Zygmund operator, then $T$ satisfies Assumption 1.6. On the other hand, it was proved in [14] that, if $T$ satisfies Assumptions 1.7 and 1.8, then $K$ satisfies (1.6). This shows that, the multilinear singular integral operators considered in $[6,5]$ satisfy the hypothesis of Theorems 1.10 .

Some multi(sub)linear maximal operators will be useful in the proof of Theorem 1.10. The first one is the operator $\mathcal{M}$ defined by

$$
\mathcal{M}\left(f_{1}, \ldots, f_{m}\right)(x)=\sup _{Q \ni x} \prod_{j=1}^{m}\left(\frac{1}{|Q|} \int_{Q}\left|f_{j}\left(y_{j}\right)\right| \mathrm{d} y_{j}\right) .
$$

This operator was introduced in [16], and plays an important role in the study of the weighted estimates with multiple weights for multilinear Calderón-Zygmund operators. Let $\Lambda$ be a non-trivial subset of $\{1, \ldots, m\}$ and $\# \Lambda$ be the cardinal number of $\Lambda$. Define the multi(sub)linear operator $\mathcal{M}_{\Lambda}$ by

$$
\begin{aligned}
\mathcal{M}_{\Lambda}\left(f_{1}, \ldots, f_{m}\right)(x)=\sup _{Q \ni x} & \sum_{l=1}^{\infty} 2^{-n l \# \Lambda} \prod_{i \in \Lambda}\left(\frac{1}{|Q|} \int_{Q}\left|f_{i}\left(y_{i}\right)\right| \mathrm{d} y_{i}\right) \\
& \times \prod_{j \notin \Lambda}\left(\frac{1}{\left|2^{l} Q\right|} \int_{2^{l} Q}\left|f_{j}\left(y_{j}\right)\right| \mathrm{d} y_{j}\right) .
\end{aligned}
$$

This operator was introduced by Grafakos, Liu and Yang [10], and used in the study of weighted norm inequalities with multiple weights for the multilinear singular integral operators with non-smooth kernels. Note that if $\Lambda \subset\{1, \ldots, m\}$ and $i \in \Lambda$, then

$$
\mathcal{M}_{\Lambda}\left(f_{1}, \ldots, f_{m}\right)(x) \lesssim \mathcal{M}_{i}\left(f_{1}, \ldots, f_{m}\right)(x)
$$


with

$$
\begin{aligned}
\mathcal{M}_{i}\left(f_{1}, \ldots, f_{m}\right)(x)= & \sup _{Q \ni x} \sum_{l=1}^{\infty} 2^{-n l}\left(\frac{1}{|Q|} \int_{Q}\left|f_{i}\left(y_{i}\right)\right| \mathrm{d} y_{i}\right) \\
& \times \prod_{\substack{1 \leq j \leq m \\
j \neq i}}\left(\frac{1}{\left|2^{l} Q\right|} \int_{2^{l} Q}\left|f_{j}\left(y_{j}\right)\right| \mathrm{d} y_{j}\right) .
\end{aligned}
$$

For the operators $\mathcal{M}$ and $\mathcal{M}_{i}$, we have

Theorem 1.13. Let $p_{1}, \ldots, p_{m} \in[1, \infty), q_{1}, \ldots, q_{m} \in(1, \infty), p, q \in(0, \infty)$ such that $1 / p=1 / p_{1}+\cdots+1 / p_{m}, 1 / q=1 / q_{1}+\cdots+1 / q_{m}, \vec{w}=\left(w_{1}, \ldots, w_{m}\right) \in A_{\vec{P}}\left(\mathbb{R}^{m n}\right)$. All of the operators $\mathcal{M}, \mathcal{M}_{i}(i=1, \ldots, m)$ are bounded from $L^{p_{1}}\left(l^{q_{1}} ; \mathbb{R}^{n}, w_{1}\right) \times$ $\cdots \times L^{p_{m}}\left(l^{q_{m}} ; \mathbb{R}^{n}, w_{m}\right)$ to $L^{p, \infty}\left(l^{q} ; \mathbb{R}^{n}, \nu_{\vec{w}}\right)$. Moreover, if $\min _{1 \leq j \leq m} p_{j}>1$, then the operators $\mathcal{M}$ and $\mathcal{M}_{i}$ are bounded from $L^{p_{1}}\left(l^{q_{1}} ; \mathbb{R}^{n}, w_{1}\right) \times \cdots \times L^{p_{m}}\left(l^{q_{m}} ; \mathbb{R}^{n}, w_{m}\right)$ to $L^{p}\left(l^{q} ; \mathbb{R}^{n}, \nu_{\vec{w}}\right)$.

In what follows, $C$ always denotes a positive constant that is independent of the main parameters involved but whose value may differ from line to line. We use the symbol $A \lesssim B$ to denote that there exists a positive constant $C$ such that $A \leq C B$. Constant with subscript such as $C_{1}$, does not change in different occurrences. For any set $E \subset \mathbb{R}^{n}, \chi_{E}$ denotes its characteristic function. For a cube $Q \subset \mathbb{R}^{n}$ and $\lambda \in(0, \infty)$, we use $\ell(Q)(\operatorname{diam} Q)$ to denote the side length (diamter) of $Q$, and $\lambda Q$ to denote the cube with the same center as $Q$ and whose side length is $\lambda$ times that of $Q$. For $x \in \mathbb{R}^{n}$ and $r>0, B(x, r)$ denotes the ball centered at $x$ and having radius $r$.

\section{Proof of Theorem 1.10}

We begin with a variant of the Whitney decomposition lemma, see [19].

Lemma 2.1. Let $R>1$. There exists a constant $C(n, R)$ such that for all open set $\Omega \subset \mathbb{R}^{n}, \Omega$ can be decomposed as $\Omega=\cup_{j} Q_{j}$, where $\left\{Q_{j}\right\}$ is a sequence of cubes with disjoint interiors, and

(i)

$$
5 R \leq \frac{\operatorname{dist}\left(Q_{j}, \mathbb{R}^{n} \backslash \Omega\right)}{\operatorname{diam} Q_{j}} \leq 15 R,
$$

(ii) $\sum_{j} \chi_{R Q_{j}}(x) \leq C_{n, R} \chi_{\Omega}(x)$.

Let $f \in L^{1}\left(\mathbb{R}^{n}\right)$ and $M f$ be the Hardy-Littlewood maximal function of $f$. Applying Lemma 2.1 to the set $\Omega=\left\{x \in \mathbb{R}^{n}: M f(x)>\lambda\right\}$, we can obtain a sequence of cubes $\left\{Q_{j}\right\}$ with disjoint interiors, such that

$$
\frac{1}{\left|Q_{j}\right|} \int_{Q_{j}}|f(y)| \mathrm{d} y>\lambda
$$

As in $\left[20\right.$, p. 19], we can verify that, for each $j$, there exists a cube $Q_{j}^{*}$ which contains a point $x_{j}$ such that $M f\left(x_{j}\right) \leq \lambda, Q_{j}^{*} \supset Q_{j}, \ell\left(Q_{j}^{*}\right)=(15 R+1) n \ell\left(Q_{j}\right)$. Therefore,

$$
\frac{1}{\left|Q_{j}\right|} \int_{Q_{j}}|f(y)| \mathrm{d} y \lesssim \frac{1}{\left|Q_{j}^{*}\right|} \int_{Q_{j}^{*}}|f(y)| \mathrm{d} y \lesssim M f\left(x_{j}\right) \lesssim \lambda .
$$

Moreover, $\sum_{j} \chi_{R Q_{j}}(x) \lesssim \chi_{\Omega}(x)$.

Lemma 2.2. Let $m \geq 2,1 \leq j \leq m, T$ be an $m$-linear operator with kernel $K$ in the sense of $(1.1), q_{1}, \ldots q_{m} \in(1, \infty)$ with $q \in(0, \infty)$ such that $1 / q=1 / q_{1}+\cdots+1 / q_{m}$. Suppose that

(i) $T$ is bounded from $L^{q_{1}}\left(\mathbb{R}^{n}\right) \times \cdots \times L^{q_{m}}\left(\mathbb{R}^{n}\right)$ to $L^{q}\left(\mathbb{R}^{n}\right)$; 
(ii) T satisfies the Assumption 1.6.

Then $T$ is bounded from $L^{1}\left(l^{q_{1}}, \mathbb{R}^{n}\right) \times \cdots \times L^{1}\left(l^{q_{m}}, \mathbb{R}^{n}\right)$ to $L^{1 / m, \infty}\left(l^{q}, \mathbb{R}^{n}\right)$.

Proof. We claim that if $p_{1}, \ldots, p_{m} \in(1, \infty), r_{1}, \ldots, r_{m} \in(1, \infty), 1 / p=1 / p_{1}+$ $\cdots+1 / p_{m}, 1 / r=1 / r_{1}+\cdots+1 / r_{m}, T$ is bounded from $L^{p_{1}}\left(l^{r_{1}}, \mathbb{R}^{n}\right) \times \cdots \times$ $L^{p_{m}}\left(l^{r_{m}}, \mathbb{R}^{n}\right)$ to $L^{p, \infty}\left(l^{r}, \mathbb{R}^{n}\right)$, then for each $1 \leq j_{0} \leq m, T$ is bounded from $L^{p_{1}}\left(l^{r_{1}}, \mathbb{R}^{n}\right) \times \cdots \times L^{p_{j_{0}-1}}\left(l^{r_{j_{0}-1}}, \mathbb{R}^{n}\right) \times L^{1}\left(l^{r_{j_{0}}}, \mathbb{R}^{n}\right) \times L^{p_{j_{0}+1}}\left(l^{r_{j_{0}}+1}, \mathbb{R}^{n}\right) \times \cdots \times$ $L^{p_{m}}\left(l^{r_{m}}, \mathbb{R}^{n}\right)$ to $L^{\varrho_{j_{0}}, \infty}\left(l^{r}, \mathbb{R}^{n}\right)$, where $1 / \varrho_{j_{0}}=\sum_{1 \leq j \leq m, j \neq j_{0}} 1 / p_{j}+1$. In fact, this is equivalent to prove that for each fixed $\lambda>0$,

$$
\begin{aligned}
& \left|\left\{x \in \mathbb{R}^{n}:\left\|\left\{T\left(f_{1}^{k}, \ldots, f_{m}^{k}\right)(x)\right\}\right\|_{l^{r}}>\lambda\right\}\right| \\
& \quad \lesssim \lambda^{-\varrho_{j_{0}}} \prod_{1 \leq j \leq m, j \neq j_{0}}\left\|\left\{f_{j}^{k}\right\}\right\|_{L^{p_{j}}\left(l^{r_{j}} ; \mathbb{R}^{n}\right)}^{\varrho_{j_{0}}}\left\|\left\{f_{m}^{k}\right\}\right\|_{L^{1}\left(l^{r_{j}} ; \mathbb{R}^{n}\right)}^{\varrho_{j_{0}}}
\end{aligned}
$$

For simplicity, we only consider the case $j_{0}=m$. By homogeneity, we may assume that

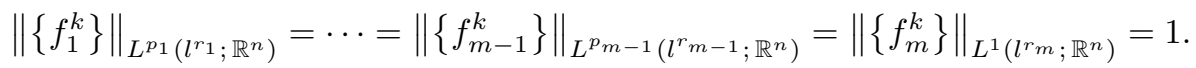

For $\lambda>0$, applying Lemma 2.1 to $\Omega_{m}=\left\{x \in \mathbb{R}^{n}: M\left(\left\|\left\{f_{m}^{k}\right\}\right\|_{l^{r_{m}}}\right)(x)>\lambda^{\varrho_{m}}\right\}$ and $R=4$, we obtain a sequence of cubes $\left\{Q_{m}^{l}\right\}$ with disjoint interiors, such that

$$
\lambda^{\varrho_{m}}<\frac{1}{\left|Q_{m}^{l}\right|} \int_{Q_{m}^{l}}\left\|\left\{f_{m}^{k}(x)\right\}\right\|_{l^{r_{m}}} \mathrm{~d} x \lesssim \lambda^{\varrho_{m}},
$$

and $\sum_{l} \chi_{4 Q_{m}^{l}}(x) \lesssim \chi_{\Omega_{m}}(x)$. For each fixed $k$, set

$$
\begin{gathered}
f_{m}^{k, 1}(x)=f_{m}^{k}(x) \chi_{\mathbb{R}^{n} \backslash \Omega_{m}}(x), \\
f_{m}^{k, 2}(x)=\sum_{l} A_{t_{Q_{m}^{l}}^{l}}^{m} b_{m}^{k, l}(x), f_{m}^{k, 3}(x)=\sum_{l}\left(b_{m}^{k, l}(x)-A_{t_{Q_{m}^{l}}}^{m} b_{m}^{k, l}(x)\right),
\end{gathered}
$$

with $b_{m}^{k, l}(y)=f_{m}^{k}(y) \chi_{Q_{m}^{l}}(y), t_{Q_{m}^{l}}=\left\{\ell\left(Q_{m}^{l}\right)\right\}^{s}$ and $s$ is the constant appeared in (1.4). Our proof is now reduced to proving that for $i=1,2,3$,

$$
\left|\left\{x \in \mathbb{R}^{n}:\left\|\left\{T\left(f_{1}^{k}, \ldots, f_{m-1}^{k}, f_{m}^{k, i}\right)(x)\right\}\right\|_{l^{r}}>\lambda / 3\right\}\right| \lesssim \lambda^{-\varrho_{m}},
$$

We first prove $(2.3)$ for $i=1,2$. By the fact that $\left\|\left\{f_{m}^{k, 1}\right\}\right\|_{L^{\infty}\left(l^{r} m ; \mathbb{R}^{n}\right)} \lesssim \lambda^{\varrho_{m}}$, we deduce that

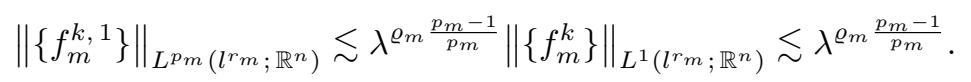

Recalling that $T$ is bounded from $L^{p_{1}}\left(l^{r_{1}} ; \mathbb{R}^{n}\right) \times \cdots \times L^{p_{m}}\left(l^{r_{m}} ; \mathbb{R}^{n}\right)$ to $L^{p, \infty}\left(l^{r} ; \mathbb{R}^{n}\right)$, and $1 / \varrho_{m}=\sum_{j=1}^{m-1} 1 / p_{j}+1$, we have by the inequality (2.4) that

$$
\begin{aligned}
& \left|\left\{x \in \mathbb{R}^{n}:\left\|\left\{T\left(f_{1}^{k}, \ldots, f_{m-1}^{k}, f_{m}^{k, 1}\right)(x)\right\}\right\|_{l^{r}}>\lambda / 3\right\}\right| \\
& \quad \lesssim \lambda^{-p}\left\|\left\{f_{m}^{k, 1}\right\}\right\|_{L^{p_{m}}\left(l^{r_{m}} ; \mathbb{R}^{n}\right)}^{p} \lesssim \lambda^{-\varrho_{m}} .
\end{aligned}
$$

To prove (2.3) for $i=2$, we first get from (1.4) and (1.5) that

$$
\begin{aligned}
\int_{\mathbb{R}^{n}}\left|v_{k}(y) A_{t_{Q_{m}^{l}}^{m}}^{m} b_{m}^{k, l}(y)\right| \mathrm{d} y & \leq \int_{Q_{m}^{l}}\left|b_{m}^{k, l}(z)\right| \int_{\mathbb{R}^{n}} h_{Q_{Q_{m}^{l}}}^{m}(z, y)\left|v_{k}(z)\right| \mathrm{d} z \mathrm{~d} y \\
& \lesssim \int_{Q_{m}^{l}}\left|b_{m}^{k, l}(z)\right| \mathrm{d} z \inf _{y \in Q_{m}^{l}} M v_{k}(y) .
\end{aligned}
$$

On the other hand, a straightforward computation involving Minkowski's inequality gives us that

$$
\left(\sum_{k}\left\|b_{m}^{k, l}\right\|_{L^{1}\left(\mathbb{R}^{n}\right)}^{r_{m}}\right)^{1 / r_{m}} \leq \int_{Q_{m}^{l}}\left(\sum_{k}\left|f_{m}^{k}(y)\right|^{r_{m}}\right)^{1 / r_{m}} d y \lesssim \lambda^{\varrho_{m}}\left|Q_{m}^{l}\right| .
$$


Therefore, by Minkowski's inequality and the vector-valued inequality of the HardyLittlewood maximal operator $M$ (see [7]),

$$
\begin{aligned}
& \left\|\left(\sum_{k}\left|\sum_{l} A_{t_{Q_{m}^{l}}^{m}} b_{m}^{k, l}\right|^{r_{m}}\right)^{1 / r_{m}}\right\|_{L^{p_{m}\left(\mathbb{R}^{n}\right)}}
\end{aligned}
$$

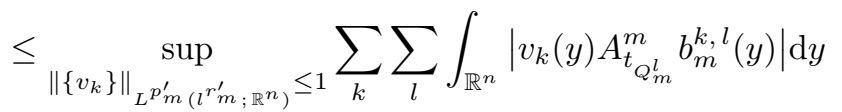

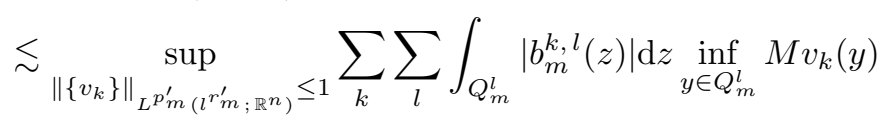

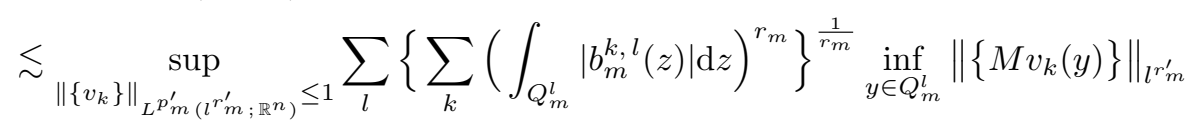

$$
\begin{aligned}
& \lesssim \sup _{\left\|\left\{v_{k}\right\}\right\|_{L^{p_{m}^{\prime}}\left(l^{\left.r_{m}^{\prime} ; \mathbb{R}^{n}\right)} \leq 1\right.}} \sum_{l} \int_{Q_{m}^{l}}\left\|\left\{b_{m}^{k, l}(z)\right\}\right\|_{l^{r_{m}}} \mathrm{~d} z \inf _{y \in Q_{m}^{l}}\left\|\left\{M v_{k}(y)\right\}\right\|_{l^{r_{m}^{\prime}}} \\
& \lesssim \lambda^{Q_{m}} \sup _{\left\|\left\{v_{k}\right\}\right\|_{L^{p_{m}^{\prime}}\left(l^{r_{m}} ; \mathbb{R}^{n}\right)} \leq 1} \int_{\mathbb{R}^{n}}\left\|\left\{M v_{k}(y)\right\}\right\|_{l^{r_{m}^{\prime}}} \sum_{l} \chi_{Q_{m}^{l}}(y) \mathrm{d} y \\
& \lesssim \lambda^{\varrho_{m} \frac{p_{m}-1}{p_{m}}} .
\end{aligned}
$$

This, along with the fact that $T$ is bounded from $L^{p_{1}}\left(l^{r_{1}} ; \mathbb{R}^{n}\right) \times \cdots \times L^{p_{m}}\left(l^{r_{m}} ; \mathbb{R}^{n}\right)$ to $L^{p, \infty}\left(l^{r} ; \mathbb{R}^{n}\right)$, leads to that

$$
\left|\left\{x \in \mathbb{R}^{n}:\left\|\left\{T\left(f_{1}^{k}, \ldots, f_{m-1}^{k}, f_{m}^{k, 2}\right)(x)\right\}\right\|_{l^{r}}>\lambda / 3\right\}\right| \lesssim \lambda^{-\varrho_{m}} .
$$

Now we prove the estimate $(2.3)$ for $i=3$. Let $\tilde{\Omega}_{m}=\cup_{l} 4 n Q_{m}^{l}$. It is obvious that $\left|\tilde{\Omega}_{m}\right| \lesssim \lambda^{-\varrho_{m}}$. Let

$$
\begin{gathered}
\mathrm{I}_{k}(x)=\sum_{l} \int_{\mathbb{R}^{m n}} \frac{\left\{\ell\left(Q_{m}^{l}\right)\right\}^{\varepsilon}}{\left(\sum_{j=1}^{m}\left|x-y_{j}\right|\right)^{m n+\varepsilon}} \prod_{j=1}^{m-1}\left|f_{j}^{k}\left(y_{j}\right)\right|\left|b_{m}^{k, l}\left(y_{m}\right)\right| \mathrm{d} \vec{y}, \\
\mathrm{II}_{k}(x)=\sum_{j=1}^{m-1} \sum_{l} \int_{\mathbb{R}^{m n}} \phi\left(\frac{\left|y_{j}-y_{m}\right|}{\ell\left(Q_{m}^{l}\right)}\right) \prod_{i=1}^{m-1}\left|f_{i}^{k}\left(y_{i}\right)\right| \frac{\left|b_{m}^{k, l}\left(y_{m}\right)\right|}{\left(\sum_{i=1}^{m}\left|x-y_{i}\right|\right)^{m n}} \mathrm{~d} \vec{y} .
\end{gathered}
$$

By Assumption 1.6, we know that for each $x \in \mathbb{R}^{n} \backslash \tilde{\Omega}_{m}$,

$$
\begin{aligned}
& \left|T\left(f_{1}^{k}, \ldots, f_{m-1}^{k}, f_{m}^{k, 3}\right)(x)\right| \\
& \leq \sum_{l} \int_{\mathbb{R}^{m n}}\left|K\left(x ; y_{1}, \ldots, y_{m}\right)-K_{A_{t} Q_{m}^{l}}^{m}\left(x ; y_{1}, \ldots, y_{m}\right)\right| \prod_{j=1}^{m-1}\left|f_{j}^{k}\left(y_{j}\right)\right| b_{m}^{k, l}\left(y_{m}\right) \mid \mathrm{d} \vec{y} \\
(2.6) \lesssim \mathrm{I}_{k}(x)+\mathrm{II}_{k}(x) . &
\end{aligned}
$$

Let $x_{m}^{l}$ be the center of $Q_{m}^{l}$, and

$$
\mathcal{N}^{k}(x)=\sum_{l} \frac{\left\{\ell\left(Q_{m}^{l}\right)\right\}^{\varepsilon}}{\left|x-x_{m}^{l}\right|^{n+\varepsilon}}\left\|b_{m}^{k, l}\right\|_{L^{1}\left(\mathbb{R}^{n}\right)} .
$$

It follows from (2.5) that

$$
\begin{aligned}
\left\|\left\{\mathcal{N}^{k}(x)\right\}\right\|_{l^{r_{m}}} & \leq \sum_{l}\left(\sum_{k}\left\|b_{m}^{k, l}\right\|_{L^{1}\left(\mathbb{R}^{n}\right)}^{r_{m}}\right)^{1 / r_{m}} \frac{\left\{\ell\left(Q_{m}^{l}\right)\right\}^{\varepsilon}}{\left|x-x_{m}^{l}\right|^{n+\varepsilon}} \\
& \lesssim \lambda^{\varrho_{m}} \sum_{l}\left|Q_{m}^{l}\right| \frac{\left\{\ell\left(Q_{m}^{l}\right)\right\}^{\varepsilon}}{\left|x-x_{m}^{l}\right|^{n+\varepsilon}} .
\end{aligned}
$$


Observing that

$$
\mathrm{I}_{k}(x) \lesssim \mathcal{N}^{k}(x) \prod_{j=1}^{m-1} M f_{j}^{k}(x)
$$

we then deduce that

$$
\begin{aligned}
\left\|\left\{\mathrm{I}_{k}(x)\right\}\right\|_{l^{r}} & \lesssim\left(\sum_{k}\left(\mathcal{N}^{k}(x)\right)^{r_{m}}\right)^{1 / r_{m}} \prod_{j=1}^{m-1}\left\|\left\{M f_{j}^{k}(x)\right\}\right\|_{l^{r_{j}}} \\
& \lesssim \lambda^{\varrho_{m}} \sum_{l} \frac{\left|Q_{m}^{l}\right|\left\{\ell\left(Q_{m}^{l}\right)\right\}^{\varepsilon}}{\left|x-x_{m}^{l}\right|^{n+\varepsilon}} \prod_{j=1}^{m-1}\left\|\left\{M f_{j}^{k}(x)\right\}\right\|_{l^{r_{j}}} .
\end{aligned}
$$

Another application of the vector-valued inequality for $M$, leads to that

$$
\begin{aligned}
\left|\left\{x \in \mathbb{R}^{n} \backslash \tilde{\Omega}_{m}:\left\|\left\{\mathrm{I}_{k}(x)\right\}\right\|_{l^{r}}>\lambda / 6\right\}\right| \lesssim & \sum_{j=1}^{m-1}\left|\left\{x \in \mathbb{R}^{n}:\left\|\left\{M f_{j}^{k}(x)\right\}\right\|_{l^{r_{j}}}>\lambda^{\frac{\varrho m}{p_{j}}}\right\}\right| \\
& +\sum_{l}\left|Q_{m}^{l}\right| \int_{\mathbb{R}^{n} \backslash \Omega_{m}} \frac{\left\{\ell\left(Q_{m}^{l}\right)\right\}^{\varepsilon}}{\left|x-x_{m}^{l}\right|^{n+\varepsilon}} \mathrm{d} x \lesssim \lambda^{-\varrho_{m}} .
\end{aligned}
$$

We turn our attention to $\left\|\left\{\mathrm{II}_{k}(x)\right\}\right\|_{l^{r}}$. For $1 \leq j \leq m-1$, set

$$
\mathrm{II}_{k}^{j}(x)=\sum_{l} \int_{\mathbb{R}^{m n}} \frac{1}{\left(\sum_{i=1}^{m}\left|x-y_{i}\right|\right)^{m n}} \phi\left(\frac{\left|y_{j}-y_{m}\right|}{\ell\left(Q_{m}^{l}\right)}\right) \prod_{i=1}^{m-1}\left|f_{i}^{k}\left(y_{i}\right)\right|\left|b_{m}^{k, l}\left(y_{m}\right)\right| \mathrm{d} \vec{y}
$$

Our goal is to prove that for each $j$ with $1 \leq j \leq m-1$,

$$
\left|\left\{x \in \mathbb{R}^{n} \backslash \tilde{\Omega}_{m}:\left\|\left\{\mathrm{II}_{k}^{j}(x)\right\}\right\|_{l^{r}}>\lambda /(6 m)\right\}\right| \lesssim \lambda^{-\varrho_{m}} .
$$

If this is true, then (2.3) with $i=3$ follows from (2.6), (2.7) and (2.8) directly.

We now prove (2.8). We consider the following two cases.

Case I. $p_{j}=1$. For $x \in \mathbb{R}^{n} \backslash \widetilde{\Omega}_{m}$, write

$$
\begin{aligned}
\mathrm{II}_{k}^{j}(x) & \lesssim \prod_{1 \leq i \leq m-1, i \neq j} M f_{i}^{k}(x) \sum_{l} \int_{\mathbb{R}^{n}} \int_{4 Q_{m}^{l}} \frac{\left|f_{j}^{k}\left(y_{j}\right)\right|\left|b_{m}^{k, l}\left(y_{m}\right)\right|}{\left|x-y_{j}\right|^{2 n}} \mathrm{~d} y_{j} \mathrm{~d} y_{m} \\
& \lesssim \prod_{1 \leq i \leq m-1, i \neq j} M f_{i}^{k}(x) \sum_{l} \frac{\left\|b_{m}^{k, l}\right\|_{L^{1}\left(\mathbb{R}^{n}\right)}}{\left|x-x_{m}^{l}\right|^{2 n}} \int_{4 Q_{m}^{l}}\left|f_{j}^{k}\left(y_{j}\right)\right| \mathrm{d} y_{j} .
\end{aligned}
$$

Let

$$
\mathrm{D}_{j, m}^{k, l}(x)=\int_{4 Q_{m}^{l}}\left|f_{j}^{k}\left(y_{j}\right)\right| \mathrm{d} y_{j}, \mathrm{E}_{j, m}^{k, l}(x)=\frac{\left\|b_{m}^{k, l}\right\|_{L^{1}\left(\mathbb{R}^{n}\right)}}{\left|x-x_{m}^{l}\right|^{2 n}},
$$

Again by Minkowski's inequality,

$$
\left\{\sum_{k}\left(\mathrm{D}_{j, m}^{k, l}(x)\right)^{r_{j}}\right\}^{1 / r_{j}} \leq \int_{4 Q_{m}^{l}}\left\|\left\{f_{j}^{k}\left(y_{j}\right)\right\}\right\|_{l^{r_{j}}} \mathrm{~d} y_{j} .
$$

On the other hand, it follows from (2.5) that

$$
\left\{\sum_{k}\left(\mathrm{E}_{j, m}^{k, l}(x)\right)^{r_{m}}\right\}^{1 / r_{m}} \lesssim \lambda^{\varrho_{m}} \frac{\left|Q_{m}^{l}\right|}{\left|x-x_{m}^{l}\right|^{2 n}}
$$

Set $\mu_{j, m} \in(0, \infty)$ such that $1 / \mu_{j, m}=1 / r_{j}+1 / r_{m}$. We can take $\nu \in(1 / 2,1)$ such that $\nu_{j, m} / \mu>1$ since $\mu_{j, m}>1 / 2$. Let

$$
\mathrm{F}_{j}^{\mu}(x)=\left\{\sum_{l} \frac{\left|Q_{m}^{l}\right|^{\mu}}{\left|x-x_{m}^{l}\right|^{2 n \mu}}\left[\int_{4 Q_{m}^{l}}\left\|\left\{f_{j}^{k}\left(y_{j}\right)\right\}\right\|_{l^{r_{j}}} \mathrm{~d} y_{j}\right]^{\mu}\right\}^{1 / \mu} .
$$


An argument involving Minowski's inequality and Hölder's inequality, (2.9) and (2.10), now tells us that

$$
\begin{aligned}
& \left(\sum_{k}\left|\sum_{l} \mathrm{E}_{j, m}^{k, l}(x) \mathrm{D}_{j, m}^{k, l}(x)\right|^{\mu_{j, m}}\right)^{\frac{1}{\mu_{j, m}}} \\
& \leq\left(\left\{\sum_{k}\left|\sum_{l}\left[\mathrm{E}_{j, m}^{k, l}(x) \mathrm{D}_{j, m}^{k, l}(x)\right]^{\mu}\right|^{\frac{\mu_{j, m}}{\mu}}\right\}^{\frac{\mu}{\mu_{j, m}}}\right)^{\frac{1}{\mu}} \\
& \quad \lesssim\left\{\sum_{l}\left(\sum_{k}\left|\mathrm{E}_{j, m}^{k, l}(x)\right|^{r_{m}}\right)^{\frac{\mu}{r_{m}}}\left(\sum_{k}\left|\mathrm{D}_{j, m}^{k, l}(x)\right|^{r_{j}}\right)^{\frac{\mu}{r_{j}}}\right\}^{\frac{1}{\mu}} \\
& \quad \lesssim \lambda^{\varrho_{m}} \mathrm{~F}_{j}^{\mu}(x),
\end{aligned}
$$

Thus, by Hölder's inequality,

$$
\left\|\left\{\mathrm{II}_{k}^{j}(x)\right\}\right\|_{l^{r}} \lesssim \lambda^{\varrho_{m}} \mathrm{~F}_{j}^{\mu}(x) \prod_{1 \leq i \leq m-1, i \neq j}\left\|\left\{M f_{i}^{k}(x)\right\}\right\|_{l^{r_{i}}} .
$$

It is easy to verify that

$$
\begin{aligned}
& \int_{\mathbb{R}^{n} \backslash \Omega_{m}}\left|\mathrm{~F}_{j}^{\mu}(x)\right|^{\mu} \mathrm{d} x \lesssim \sum_{l}\left|Q_{m}^{l}\right|^{-\mu+1}\left(\int_{4 Q_{m}^{l}}\left\|\left\{f_{j}^{k}\left(y_{j}\right)\right\}\right\|_{l^{r_{j}}} \mathrm{~d} y_{j}\right)^{\mu} \\
& \lesssim\left(\sum_{l}\left|Q_{m}^{l}\right|\right)^{1-\mu}\left(\sum_{u} \int_{4 Q_{m}^{u}}\left\|\left\{f_{j}^{k}\left(y_{j}\right)\right\}\right\|_{l^{r_{j}}} \mathrm{~d} y_{j}\right)^{\mu} \\
& \lesssim \lambda^{-\varrho_{m}(1-\mu)}\left\|\left\{f_{j}^{k}\right\}\right\|_{L^{1}\left(l^{\left.r_{j} ; \mathbb{R}^{n}\right)}\right.}^{\mu} .
\end{aligned}
$$

Recall that $p_{j}=1$. We obtain from (2.11) and (2.12) and the vector-valued inequality for the operator $M$ that

$$
\begin{aligned}
& \left|\left\{x \in \mathbb{R}^{n} \backslash \tilde{\Omega}_{m}:\left\|\left\{\mathrm{II}_{k}^{j}(x)\right\}\right\|_{l^{r}}>\lambda /(6 m)\right\}\right| \lesssim \lambda^{-\varrho_{m} \mu} \int_{\mathbb{R}^{n} \backslash \tilde{\Omega}_{m}}\left|\mathrm{~F}_{j}^{\mu}(x)\right|^{\mu} \mathrm{d} x \\
& \quad+\sum_{1 \leq i \leq m, i \neq j}\left|\left\{x \in \mathbb{R}^{n}:\left\|\left\{M f_{i}^{k}(x)\right\}\right\|_{l^{r_{i}}}>\lambda^{\frac{\varrho m}{p_{i}}} /(6 m)\right\}\right| \\
& \quad \lesssim \lambda^{-\varrho_{m}} .
\end{aligned}
$$

Case II $p_{j} \in(1, \infty)$. We take $\sigma \in\left(1, \min \left\{p_{j}, r_{j}\right\}\right)$. Set

$$
\mathrm{G}(x)=\sum_{l} \frac{\left|Q_{m}^{l}\right|^{2-1 / \sigma}}{\left|x-x_{m}^{l}\right|^{2 n-n / \sigma}} .
$$

It is easy to verify that

$$
\int_{\mathbb{R}^{n} \backslash \tilde{\Omega}_{m}} \mathrm{G}(x) \mathrm{d} x \lesssim \sum_{l}\left|Q_{m}^{l}\right| \lesssim \lambda^{-\varrho_{m}} .
$$

For $x \in \mathbb{R}^{n} \backslash \tilde{\Omega}$, it is obvious that $4 Q_{m}^{l} \subset B\left(x, 2\left|x-x_{m}^{l}\right|\right)$ and so we have

$$
\begin{aligned}
\int_{4 Q_{m}^{l}}\left|f_{j}^{k}\left(y_{j}\right)\right| \mathrm{d} y_{j} & \lesssim\left|Q_{m}^{l}\right|^{1-\frac{1}{\sigma}}\left(\int_{4 Q_{m}^{l}}\left|f_{j}^{k}\left(y_{j}\right)\right|^{\sigma} \mathrm{d} y_{j}\right)^{\frac{1}{\sigma}} \\
& \lesssim\left|Q_{m}^{l}\right|^{1-\frac{1}{\sigma}}\left|x-x_{m}^{l}\right|^{\frac{1}{\sigma}} M_{\sigma} f_{j}^{k}(x),
\end{aligned}
$$

where and in the following, $M_{\sigma} f(x)=\left[M\left(|f|^{\sigma}\right)(x)\right]^{1 / \sigma}$. It then follows from Hölder's inequality that when $x \in \mathbb{R}^{n} \backslash \tilde{\Omega}$,

$$
\mathrm{II}_{k}^{j}(x) \lesssim \prod_{1 \leq i \leq m-1, i \neq j} M f_{i}^{k}(x) \sum_{l} \frac{\left\|b_{m}^{k, l}\right\|_{L^{1}\left(\mathbb{R}^{n}\right)}}{\left|x-x_{m}^{l}\right|^{2 n}} \int_{4 Q_{m}^{l}}\left|f_{j}^{k}\left(y_{j}\right)\right| \mathrm{d} y_{j}
$$




$$
\lesssim \prod_{1 \leq i \leq m-1, i \neq j} M f_{i}^{k}(x) M_{\sigma} f_{j}^{k}(x) \sum_{l} \frac{\left\|b_{m}^{k, l}\right\|_{L^{1}\left(\mathbb{R}^{n}\right)}}{\left|x-x_{m}^{l}\right|^{2 n-n / \sigma}}\left|Q_{m}^{l}\right|^{1-1 / \sigma} .
$$

This, together Hölder's inequality and Minkowski's inequality and the estimate (2.6), implies that

$$
\left\|\left\{\mathrm{II}_{k}^{j}(x)\right\}\right\|_{l^{r}} \lesssim \lambda^{\varrho_{m}}\left\|\left\{M_{\sigma}\left(f_{j}^{k}\right)(x)\right\}\right\|_{l^{r_{j}}} G(x) \prod_{1 \leq i \leq m-1, i \neq j}\left\|\left\{M f_{i}^{k}(x)\right\}\right\|_{l^{r_{i}}} .
$$

Therefore,

$$
\begin{aligned}
& \left|\left\{x \in \mathbb{R}^{n} \backslash \Omega_{m}:\left\|\left\{\mathrm{II}_{k}^{j}(x)\right\}\right\|_{l^{r}}>\lambda /(6 m)\right\}\right| \\
& \quad \lesssim \int_{\mathbb{R}^{n} \backslash \Omega_{m}} \mathrm{G}(x) \mathrm{d} x+\left|\left\{x \in \mathbb{R}^{n}:\left\|\left\{M_{\sigma}\left(f_{j}^{k}\right)(x)\right\}\right\|_{l^{r_{j}}}>C_{m} \lambda^{\frac{\underline{\underline{m}}}{p_{j}}}\right\}\right| \\
& \quad+\sum_{1 \leq i \leq m, i \neq j}\left|\left\{x \in \mathbb{R}^{n}:\left\|\left\{M f_{i}^{k}(x)\right\}\right\|_{l^{r_{i}}}>C_{m} \lambda^{\frac{\varrho \underline{\rho_{m}}}{p_{i}}}\right\}\right| \\
& \quad \lesssim \lambda^{-\varrho_{m}} .
\end{aligned}
$$

We can now conclude the proof of Lemma 2.2. The assumption (i) tells us that $T$ is bounded from $L^{q_{1}}\left(l^{q_{1}}, \mathbb{R}^{n}\right) \times \cdots \times L^{q_{m}}\left(l^{q_{m}}, \mathbb{R}^{n}\right)$ to $L^{q}\left(l^{q}, \mathbb{R}^{n}\right)$. Thus, by our claim, $T$ is bounded from $L^{q_{1}}\left(l^{q_{1}}, \mathbb{R}^{n}\right) \times \cdots \times L^{q_{m-1}}\left(l^{q_{m-1}}, \mathbb{R}^{n}\right) \times L^{1}\left(l^{q_{m}}, \mathbb{R}^{n}\right)$ to $L^{\tilde{q}_{m}, \infty}\left(l^{q}, \mathbb{R}^{n}\right)$ with $1 / \tilde{q}_{m}=\sum_{1 \leq j \leq m-1} 1 / q_{j}+1$. Another application of our claim shows that $T$ is bounded from $L^{q_{1}}\left(l^{q_{1}}, \mathbb{R}^{n}\right) \times \cdots \times L^{q_{m-2}}\left(l^{q_{m-2}}, \mathbb{R}^{n}\right) \times L^{1}\left(l^{q_{m-1}}, \mathbb{R}^{n}\right) \times$ $L^{1}\left(l^{q_{m}}, \mathbb{R}^{n}\right)$ to $L^{\tilde{q}_{m-1}}\left(l^{q}, \mathbb{R}^{n}\right)$ with $1 / \tilde{q}_{m-1}=\sum_{1 \leq j \leq m-2} 1 / q_{j}+2$. Repeating the argument above $m$ times then yields the desired conclusion.

As in the proof of Kolmogrov's inequality ([8, p. 485]), we deduce from Lemma 2.2 that

Corollary 2.13. Let $\delta \in(0,1 / m)$. Under the hypothesis of Lemma 2.2, for any cube $Q \subset \mathbb{R}^{n}$,

$$
\left(\frac{1}{|Q|} \int_{Q}\left\|\left\{T\left(f_{1}^{k}, \ldots, f_{m}^{k}\right)(y)\right\}\right\|_{l^{q}}^{\delta} \mathrm{d} y\right)^{1 / \delta} \lesssim \prod_{j=1}^{m}\left(\frac{1}{|Q|} \int_{\mathbb{R}^{n}}\left\|\left\{f_{j}^{k}\left(y_{j}\right)\right\}\right\|_{l^{q_{j}}} \mathrm{~d} y_{j}\right) .
$$

Let $1 \leq i \leq m$ and $l \in \mathbb{N}$, define the operator $\mathcal{M}_{i}^{l}$ by

$$
\mathcal{M}_{i}^{l}\left(f_{1}, \ldots, f_{m}\right)(x)=\sup _{Q \ni x}\left(\frac{1}{|Q|} \int_{Q}\left|f_{i}\left(y_{i}\right)\right| \mathrm{d} y_{i}\right) \prod_{1 \leq j \leq m, j \neq i}\left(\frac{1}{\left|2^{l} Q\right|} \int_{2^{l} Q}\left|f_{j}\left(y_{j}\right)\right| \mathrm{d} y_{j}\right) .
$$

It is easy to verify that

$$
\mathcal{M}_{i}\left(f_{1}, \ldots, f_{m}\right)(x) \leq \sum_{l=1}^{\infty} 2^{-n l} \mathcal{M}_{i}^{l}\left(f_{1}, \ldots, f_{m}\right)(x)
$$

Let $M_{\nu_{\vec{w}}}^{c}$ be the weighted centered maximal operator with respected to $\nu_{\vec{w}}$, defined as

$$
M_{\nu_{\vec{w}}}^{c} f(x)=\sup _{r>0} \frac{1}{\nu_{\vec{w}}(B(x, r))} \int_{B(x, r)}|f(y)| \nu_{\vec{w}}(y) \mathrm{d} y .
$$

It is well know that $M_{\nu_{\vec{w}}}^{c}$ is bounded from $L^{1}\left(\mathbb{R}^{n}, \nu_{\vec{w}}\right)$ to $L^{1, \infty}\left(\mathbb{R}^{n}, \nu_{\vec{w}}\right)$, and bounded from $L^{p}\left(\mathbb{R}^{n}, \nu_{\vec{w}}\right)$ to $L^{p}\left(\mathbb{R}^{n}, \nu_{\vec{w}}\right)$ for $p \in(1, \infty]$.

Lemma 2.14. Let $p_{1}, \ldots, p_{m} \in[1, \infty)$ and $p \in(0, \infty)$ with $1 / p=1 / p_{1}+\cdots+$ $1 / p_{m}, \vec{w}=\left(w_{1}, \ldots, w_{m}\right) \in A_{\vec{P}}\left(\mathbb{R}^{m n}\right)$. Then there exists a constant $\theta \in(0,1)$ such that for any $l \in \mathbb{N}$ and $1 \leq i \leq m$,

$$
\mathcal{M}_{i}^{l}\left(f_{1}, \ldots, f_{m}\right)(x) \leq C 2^{n l} 2^{-\theta l} \prod_{j=1}^{m}\left\{M_{\nu_{\vec{w}}}\left[\left|f_{j}\right|^{p_{j}} w_{j} / \nu_{\vec{w}}\right](x)\right\}^{\frac{1}{p_{j}}}
$$


with $C$ a constant independent of $i$ and $l$. Moreover, if $\min _{1 \leq j \leq m} p_{j}>1$, then there exists a constant $r>1$, such that

$$
\mathcal{M}_{i}^{l}\left(f_{1}, \ldots, f_{m}\right)(x) \lesssim 2^{n l} 2^{-\theta l} \prod_{j=1}^{m}\left\{M_{\nu_{\vec{w}}}\left[\left|f_{j}\right|^{p_{j}} w_{j} / \nu_{\vec{w}}\right]^{r}(x)\right\}^{\frac{1}{r p_{j}}}
$$

Lemma 2.14 was essentially given in the proof of Proposition 2.1 in [10].

Let $M^{\sharp}$ be the Fefferman-Stein sharp maximal operator, that is,

$$
M^{\sharp} f(x)=\sup _{Q \ni x} \inf _{c \in \mathbb{C}} \frac{1}{|Q|} \int_{Q}|f(y)-c| \mathrm{d} y .
$$

For $\delta \in(0,1]$, let $M_{\delta}^{\sharp}$ be the operator defined by $M_{\delta}^{\sharp} f(x)=\left[M^{\sharp}\left(|f|^{\delta}\right)(x)\right]^{1 / \delta}$.

Lemma 2.15. Let $\delta \in(0,1 / m)$. Under the hypothesis of Theorem 1.10, the estimate

$$
\begin{aligned}
M_{\delta}^{\sharp}\left(\|\left\{T\left(f_{1}^{k}, \ldots, f_{m}^{k}\right\} \|_{l^{r}}\right)(x)\right. & \lesssim \mathcal{M}\left(\left\|\left\{f_{1}^{k}\right\}\right\|_{l^{r_{1}}}, \ldots,\left\|\left\{f_{m}^{k}\right\}\right\|_{l^{r_{m}}}\right)(x) \\
& +\sum_{1 \leq i \leq m} \mathcal{M}_{i ; r}\left(\left\|\left\{f_{1}^{k}\right\}\right\|_{l^{r_{1}}}, \ldots,\left\|\left\{f_{m}^{k}\right\}\right\|_{l^{r_{m}}}\right)(x)
\end{aligned}
$$

holds true for finite sequences $\left\{f_{1}^{k}\right\}, \ldots,\left\{f_{m}^{k}\right\}$, where and in the following, for $r \in[1, \infty), \mathcal{M}_{i ; r}\left(h_{1}, \ldots, h_{m}\right)(x)=\mathcal{M}_{i}\left(h_{1}, \ldots, h_{m}\right)(x)$; while for $r \in(0,1)$,

$$
\mathcal{M}_{i ; r}\left(h_{1}, \ldots, h_{m}\right)(x)=\left\{\sum_{l=1}^{\infty} 2^{-n l r}\left(\mathcal{M}_{i}^{l}\left(h_{1}, \ldots, h_{m}\right)(x)\right)^{r}\right\}^{\frac{1}{r}} .
$$

Proof. For each fixed $x \in \mathbb{R}^{n}$, cube $Q$ containing $x$ and $\left\{f_{1}^{k}\right\}, \ldots,\left\{f_{m}^{k}\right\}$, decompose $f_{j}^{k}$ as

$$
f_{j}^{k}(y)=f_{j}^{k}(y) \chi_{8 n Q}(y)+f_{j}^{k}(y) \chi_{\mathbb{R}^{n} \backslash 8 n Q}(y)=: f_{j}^{k, 1}(y)+f_{j}^{k, 2}(y) .
$$

Let $y_{Q} \in Q$ such that $\sum_{k}\left|T\left(f_{1}^{k}, \ldots, f_{m}^{k}\right)\left(y_{Q}\right)\right|^{r}<\infty$. Observing that $\delta / r<1$, we then get

$$
\begin{aligned}
& \left|\left\|\left\{T\left(f_{1}^{k}, \ldots, f_{m}^{k}\right)(y)\right\}\right\|_{l^{r}}^{\delta}-\left\|\left\{T\left(f_{1}^{k, 2}, \ldots, f_{m}^{k, 2}\right)\left(y_{Q}\right)\right\}\right\|_{l^{r}}^{\delta}\right| \\
& \quad \leq\left.\left|\sum_{k}\right| T\left(f_{1}^{k}, \ldots, f_{m}^{k}\right)(y)\right|^{r}-\left.\sum_{k}\left|T\left(f_{1}^{k, 2}, \ldots, f_{m}^{k, 2}\right)\left(y_{Q}\right)\right|^{r}\right|^{\delta / r} \\
& \quad \leq\left\|\left\{T\left(f_{1}^{k}, \ldots, f_{m}^{k}\right)(y)-T\left(f_{1}^{k, 2}, \ldots, f_{m}^{k, 2}\right)\left(y_{Q}\right)\right\}\right\|_{l^{r}}^{\delta}
\end{aligned}
$$

if $r \in(0,1]$. On the other hand, it is obvious that

$$
\begin{gathered}
\|\|\left\{T\left(f_{1}^{k}, \ldots, f_{m}^{k}\right)(y)\right\}\left\|_{l^{r}}^{\delta}-\right\|\left\{T\left(f_{1}^{k, 2}, \ldots, f_{m}^{k, 2}\right)\left(y_{Q}\right)\right\} \|_{l^{r}}^{\delta} \mid \\
\leq\left\|\left\{T\left(f_{1}^{k}, \ldots, f_{m}^{k}\right)(y)-T\left(f_{1}^{k, 2}, \ldots, f_{m}^{k, 2}\right)\left(y_{Q}\right)\right\}\right\|_{l^{r}}^{\delta}
\end{gathered}
$$

holds true when $r \in(1, \infty)$. Therefore, we can write

$$
\begin{aligned}
& \frac{1}{|Q|} \int_{Q}\left|\left\|\left\{T\left(f_{1}^{k}, \ldots, f_{m}^{k}\right)(y)\right\}\right\|_{l^{r}}^{\delta}-\left\|\left\{T\left(f_{1}^{k, 2}, \ldots, f_{m}^{k, 2}\right)\left(y_{Q}\right)\right\}\right\|_{l^{r}}^{\delta}\right| \mathrm{d} y \\
& \lesssim \frac{1}{|Q|} \int_{Q}\left\|\left\{T\left(f_{1}^{k, 1}, \ldots, f_{m}^{k, 1}\right)(y)\right\}\right\|_{l^{r}}^{\delta} \mathrm{d} y \\
& \quad+\frac{1}{|Q|} \sum^{*} \int_{Q}\left\|\left\{T\left(f_{1}^{k, i_{1}}, \ldots, f_{m}^{k, i_{m}}\right)(y)\right\}\right\|_{l^{r}}^{\delta} \mathrm{d} y \\
& \quad+\frac{1}{|Q|} \int_{Q}\left\|\left\{T\left(f_{1}^{k, 2}, \ldots, f_{m}^{k, 2}\right)(y)-T\left(f_{1}^{k, 2}, \ldots, f_{m}^{k, 2}\right)\left(y_{Q}\right)\right\}\right\|_{l^{r}}^{\delta} \mathrm{d} y
\end{aligned}
$$




$$
=: \mathrm{I}_{1}+\mathrm{I}_{2}+\mathrm{I}_{3}
$$

where for each term in the sum $\sum^{*},\left\{i_{1}, \ldots, i_{m}\right\} \subset\{1,2\}$ and at least one $i_{j}=2$ and one $i_{u}=1$ with $1 \leq j, u \leq m$. We have by Corollary 2.13 that

$$
\begin{aligned}
\mathrm{I}_{1}^{1 / \delta} & \lesssim \prod_{j=1}^{m}\left(\frac{1}{|Q|} \int_{4 n Q}\left\|\left\{f_{j}^{k}\left(y_{j}\right)\right\}\right\|_{l^{r_{j}}} \mathrm{~d} y_{j}\right) \\
& \lesssim \mathcal{M}\left(\left\|\left\{f_{1}^{k}\right\}\right\|_{l^{r_{1}}}, \ldots,\left\|\left\{f_{m}^{k}\right\}\right\|_{l^{r_{m}}}\right)(x) .
\end{aligned}
$$

For $\left\{i_{1}, \ldots, i_{m}\right\} \subset\{1,2\}$ with at least one $i_{j_{0}}=1$ and $i_{u}=2\left(1 \leq j_{0}, u \leq m\right)$, set $\Lambda_{i_{1}, \ldots, i_{m}}=\left\{j: 1 \leq j \leq m, i_{j}=1\right\}$. We assume that $j_{0} \in \Lambda_{i_{1}, \ldots, i_{m}}$. For each $y \in Q$, we have by the size condition (1.2) that

$$
\begin{aligned}
\left|T\left(f_{1}^{k, i_{1}}, \ldots, f_{m}^{k, i_{m}}\right)(y)\right| \lesssim & \prod_{u \in \Lambda_{i_{1}, \ldots, i_{m}}} \int_{8 n Q}\left|f_{u}^{k}\left(y_{u}\right)\right| \mathrm{d} y_{u} \\
& \times \prod_{\substack{j \notin \Lambda_{i_{1}, \ldots, i_{m}} \\
\mathbb{R}^{n} \backslash 8 n Q}} \frac{\left|f_{j}^{k}\left(y_{j}\right)\right|}{\left.\left|y-y_{j}\right|\right)^{\# n_{i_{1}}, \ldots, i_{m}}} \mathrm{~d} y_{j} \\
& \lesssim \sum_{l=4}^{\infty} 2^{-n l}\left(\frac{1}{|8 n Q|} \int_{8 n Q}\left|f_{j_{0}}^{k}\left(y_{j_{0}}\right)\right| \mathrm{d} y_{j_{0}}\right) \\
& \times \prod_{\substack{1 \leq j \leq m \\
j \neq j_{0}}}\left(\frac{1}{\left|2^{l} n Q\right|} \int_{2^{l} n Q}\left|f_{j}^{k}\left(y_{j}\right)\right| \mathrm{d} y_{j}\right) .
\end{aligned}
$$

This, along with Hölder's inequality and Minkowski's inequality, shows that when $r \in[1, \infty)$,

$$
\begin{aligned}
\left\|\left\{T\left(f_{1}^{k, i_{1}}, \ldots, f_{m}^{k, i_{m}}\right)\right\}\right\|_{l^{r}} \lesssim & \sum_{l=4}^{\infty} 2^{-n l}\left\{\sum_{k}\left(\frac{1}{|8 n Q|} \int_{8 n Q}\left|f_{j_{0}}^{k}\left(y_{j_{0}}\right)\right| \mathrm{d} y_{j_{0}}\right)^{r_{j_{0}}}\right\}^{\frac{1}{r_{j_{0}}}} \\
& \times \prod_{\substack{1 \leq j \leq m \\
j \neq j_{0}}}\left\{\sum_{k}\left(\frac{1}{\left|2^{l} n Q\right|} \int_{2^{l} n Q}\left|f_{j}^{k}\left(y_{j}\right)\right| \mathrm{d} y_{j}\right)^{r_{j}}\right\}^{\frac{1}{r_{j}}} \\
& \lesssim \mathcal{M}_{j_{0}}\left(\left\|\left\{f_{1}^{k}\right\}\right\|_{l^{r_{1}}}, \ldots,\left\|\left\{f_{m}^{k}\right\}\right\|_{l^{r_{m}}}\right)(x) .
\end{aligned}
$$

On the other hand, if $r \in(0,1]$, we then get from (2.17) and Minkowski's inequality that

$$
\begin{aligned}
& \sum_{k}\left|T\left(f_{1}^{k, i_{1}}, \ldots, f_{m}^{k, i_{m}}\right)(y)\right|^{r} \\
& \lesssim \sum_{l=4}^{\infty} 2^{-n l r}\left\{\sum_{k}\left(\frac{1}{|8 n Q|} \int_{8 n Q}\left|f_{j_{0}}^{k}\left(y_{j_{0}}\right)\right| \mathrm{d} y_{j_{0}}\right)^{r_{j_{0}}}\right\}^{r / r_{j_{0}}} \\
& \quad \times \prod_{\substack{1 \leq j \leq m \\
j \neq j_{0}}}\left\{\sum_{k}\left(\frac{1}{\left|2^{l} n Q\right|} \int_{2^{l} n Q}\left|f_{j}^{k}\left(y_{j}\right)\right| \mathrm{d} y_{j}\right)^{r_{j}}\right\}^{r / r_{j}} \\
& \lesssim \sum_{l=4}^{\infty} 2^{-n l r}\left\{\mathcal{M}_{j_{0}}^{l}\left(\left\|\left\{f_{1}^{k}\right\}\right\|_{l^{r_{1}}}, \ldots,\left\|\left\{f_{m}^{k}\right\}\right\|_{l^{r_{m}}}\right)(x)\right\}^{r} .
\end{aligned}
$$

Therefore,

$$
\mathrm{I}_{2}^{1 / \delta} \lesssim \sum_{i=1}^{\infty} \mathcal{M}_{i ; r}\left(\left\|\left\{f_{1}^{k}\right\}\right\|_{l^{r_{1}}}, \ldots,\left\|\left\{f_{m}^{k}\right\}\right\|_{l^{r_{m}}}\right)(x)
$$


It remains to estimate $\mathrm{I}_{3}$. Note that if $y \in Q$ and $\left(y_{1}, \ldots, y_{m}\right) \in\left(\mathbb{R}^{n} \backslash 8 n Q\right)^{m n}$, then $\left|y-y_{Q}\right| \leq 2 \sqrt{n} \ell(Q)$ and $4 \sqrt{n} \ell(Q) \leq \min _{1 \leq j \leq m}\left|x-y_{j}\right|$. Thus by (1.6),

$$
\begin{aligned}
& \left|T\left(f_{1}^{k, 2}, \ldots, f_{m}^{k, 2}\right)(y)-T\left(f_{1}^{k, 2}, \ldots, f_{m}^{k, 2}\right)\left(y_{Q}\right)\right| \\
& \quad \lesssim \int_{\left(\mathbb{R}^{n} \backslash 8 n Q\right)^{m}} \frac{\{\ell(Q)\}^{\gamma}}{\left(\sum_{u=1}^{m}\left|x-y_{u}\right|\right)^{n m+\gamma}} \prod_{j=1}^{m}\left|f_{j}^{k}\left(y_{j}\right)\right| \mathrm{d} \vec{y} \\
& \quad \lesssim \sum_{l=3}^{\infty} 2^{-\gamma} \prod_{j=1}^{m}\left(\frac{1}{\left|2^{l} n Q\right|} \int_{2^{l} n Q}\left|f_{j}^{k}\left(y_{j}\right)\right| \mathrm{d} y_{j}\right) .
\end{aligned}
$$

This, along with Hölder's inequality and Minkowski's inequality, implies that

$$
\begin{aligned}
& \left\{\sum_{k}\left|T\left(f_{1}^{k, 2}, \ldots, f_{m}^{k, 2}\right)(y)-T\left(f_{1}^{k, 2}, \ldots, f_{m}^{k, 2}\right)\left(y_{Q}\right)\right|^{r}\right\}^{1 / r} \\
& \quad \lesssim \sum_{l=3}^{\infty} 2^{-\gamma} \prod_{j=1}^{m} \frac{1}{\left|2^{l} n Q\right|} \int_{2^{l} n Q}\left\|\left\{f_{j}^{k}\left(y_{j}\right)\right\}\right\|_{l^{r_{j}}} \mathrm{~d} y_{j} \\
& \quad \lesssim \mathcal{M}\left(\left\|\left\{f_{1}^{k}\right\}\right\|_{l^{r_{1}}}, \ldots,\left\|\left\{f_{m}^{k}\right\}\right\|_{l^{r_{m}}}\right)(x)
\end{aligned}
$$

if $r \in(1, \infty)$, and

$$
\begin{aligned}
& \left\{\sum_{k}\left|T\left(f_{1}^{k, 2}, \ldots, f_{m}^{k, 2}\right)(y)-T\left(f_{1}^{k, 2}, \ldots, f_{m}^{k, 2}\right)\left(y_{Q}\right)\right|^{r}\right\}^{1 / r} \\
& \quad \lesssim\left\{\sum_{l=3}^{\infty} 2^{-\gamma r} \prod_{j=1}^{m}\left[\frac{1}{\left|2^{l} n Q\right|} \int_{2^{l} n Q}\left\|\left\{f_{j}^{k}\left(y_{j}\right)\right\}\right\|_{l^{r_{j}}} \mathrm{~d} y_{j}\right]^{r}\right\}^{1 / r} \\
& \quad \lesssim \mathcal{M}\left(\left\|\left\{f_{1}^{k}\right\}\right\|_{l^{r_{1}}}, \ldots,\left\|\left\{f_{m}^{k}\right\}\right\|_{l^{r_{m}}}\right)(x)
\end{aligned}
$$

if $r \in(0,1)$. Combining the estimates for $\mathrm{I}_{1}, \mathrm{I}_{2}$ and $\mathrm{I}_{3}$ leads to $(2.16)$ and then completes the proof of Lemma 2.15 .

Proof of Theorem 1.10. Let $p_{1}, \ldots, p_{m} \in[1, \infty), p \in(0, \infty)$ with $1 / p=1 / p_{1}+$ $\cdots+1 / p_{m}$, and $\vec{w}=\left(w_{1}, \ldots, w_{m}\right) \in A_{\vec{P}}\left(\mathbb{R}^{m n}\right)$. We claim that if $r \in(0,1)$, then for each $i=1, \ldots, m$,

$$
\left\|\mathcal{M}_{i, r}\left(h_{1}, \ldots, h_{m}\right)\right\|_{L^{p, \infty}\left(\mathbb{R}^{n}, \nu_{\vec{w}}\right)} \lesssim \prod_{j=1}^{m}\left\|f_{j}\right\|_{L^{p_{j}\left(\mathbb{R}^{n}, w_{j}\right)}} .
$$

Moreover if $r \in(0,1)$ and $\min _{1 \leq j \leq m} p_{j}>1$, then

$$
\left\|\mathcal{M}_{i, r}\left(h_{1}, \ldots, h_{m}\right)\right\|_{L^{p}\left(\mathbb{R}^{n}, \nu_{\vec{w}}\right)} \lesssim \prod_{j=1}^{m}\left\|h_{j}\right\|_{L^{p}\left(\mathbb{R}^{n}, w_{j}\right)} .
$$

In fact, the estimates (2.18) follows from the fact that for some constant $C_{\theta}$ depending only on $\theta$ appeared in Lemma 2.14,

$$
\begin{aligned}
\{x & \left.\in \mathbb{R}^{n}: \mathcal{M}_{i, r}\left(h_{1}, \ldots, h_{m}\right)(x)>C_{\theta} \lambda\right\} \\
& \subset \bigcup_{l=1}^{\infty}\left\{x \in \mathbb{R}^{n}: \mathcal{M}_{i}^{l}\left(h_{1}, \ldots, h_{m}\right)(x)>2^{n l} 2^{-\theta l / 2} \lambda\right\},
\end{aligned}
$$

and Lemma 2.14. To prove 2.19, we deduce from Lemma 2.14 that for $r \in(0,1)$ and $p \in(0, r]$,

$$
\left\|\mathcal{M}_{i, r}\left(h_{1}, \ldots, h_{m}\right)\right\|_{L^{p}\left(\mathbb{R}^{n}, \nu_{\vec{w}}\right)}^{p} \lesssim \sum_{l=1}^{\infty} 2^{-n l p}\left\|\mathcal{M}_{i}^{l}\left(h_{1}, \ldots, h_{m}\right)\right\|_{L^{p}\left(\mathbb{R}^{n}, \nu_{\vec{w}}\right)}^{p}
$$




$$
\lesssim \prod_{j=1}^{m}\left\|h_{j}\right\|_{L^{p}\left(\mathbb{R}^{n} w_{j}\right)}^{p}, \text { if } \min _{1 \leq j \leq m} p_{j}>1
$$

On the other hand, for the case of $r \in(0,1)$ and $p \in(r, \infty)$, we have by Minkowski's inequality that

$$
\begin{aligned}
\left\|\mathcal{M}_{i, r}\left(h_{1}, \ldots, h_{m}\right)\right\|_{L^{p}\left(\mathbb{R}^{n}, \nu_{\vec{w}}\right)}^{r} & \lesssim \sum_{l=1}^{\infty} 2^{-n l r}\left\|\mathcal{M}_{i}^{l}\left(h_{1}, \ldots, h_{m}\right)\right\|_{L^{p}\left(\mathbb{R}^{n}, \nu_{\vec{w}}\right)}^{r} \\
& \lesssim \prod_{j=1}^{m}\left\|h_{j}\right\|_{L^{p}\left(\mathbb{R}^{n} w_{j}\right)}^{r}, \text { if } \min _{1 \leq j \leq m} p_{j}>1 .
\end{aligned}
$$

We now prove Theorem 1.10. By a standard limit argument, it suffices to consider the case that $\left\{f_{1}^{k}\right\}, \ldots,\left\{f_{m}^{k}\right\}$ are finite sequences. By Lemma 2.2 and Lemma 2.15, we know that for all $q_{1}, \ldots, q_{m} \in(1, \infty)$ and $q \in(0, \infty)$ with $1 / q=1 / q_{1}+\cdots+$ $1 / q_{m}, T$ is bounded from $L^{q_{1}}\left(\mathbb{R}^{n}\right) \times \cdots \times L^{q_{m}}\left(\mathbb{R}^{n}\right)$ to $L^{q}\left(\mathbb{R}^{n}\right)$. Again by Lemma 2.2 and Lemma 2.15, we have the estimate

$$
\begin{aligned}
& M_{\delta}^{\sharp}\left(\left\|\left\{T\left(f_{1}^{k}, \ldots, f_{m}^{k}\right)\right\}\right\|_{l^{q}}\right)(x) \lesssim \mathcal{M}\left(\left\|\left\{f_{1}^{k}\right\}\right\|_{l^{q_{1}}}, \ldots,\left\|\left\{f_{m}^{k}\right\}\right\|_{l^{q_{m}}}\right)(x) \\
& \quad+\sum_{i=1}^{m} \mathcal{M}_{i, q}\left(\left\|\left\{f_{1}^{k}\right\}\right\|_{l^{q_{1}}}, \ldots,\left\|\left\{f_{m}^{k}\right\}\right\|_{l^{q_{m}}}\right)(x),
\end{aligned}
$$

with $\delta \in(0,1 / m)$. Let $p_{1}, \ldots, p_{m} \in[1, \infty), p \in(0, \infty)$ with $1 / p=1 / p_{1}+\cdots+$ $1 / p_{m}$, and $\vec{w}=\left(w_{1}, \ldots, w_{m}\right) \in A_{\vec{P}}\left(\mathbb{R}^{m n}\right)$, By Theorem 3.7 in [16], Proposition 2.1 in [10], and the estimates (2.18) and (2.19), the maximal operators $\mathcal{M}$ and $\mathcal{M}_{i, q}$ are bounded from $L^{p_{1}}\left(\mathbb{R}^{n}, w_{1}\right) \times \cdots \times L^{p_{m}}\left(\mathbb{R}^{n}, w_{m}\right)$ to $L^{p, \infty}\left(\mathbb{R}^{n}, \nu_{\vec{w}}\right)$. Moreover, if $\max _{1 \leq j \leq m} p_{j}>1$, then these maximal operators are bounded from $L^{p_{1}}\left(\mathbb{R}^{n}, w_{1}\right) \times$ $\cdots \times L^{p_{m}}\left(\mathbb{R}^{n}, w_{m}\right)$ to $L^{p}\left(\mathbb{R}^{n}, \nu_{\vec{w}}\right)$. Noticing that $\nu_{\vec{w}} \in A_{p / \delta}\left(\mathbb{R}^{n}\right)$, we then obtain the desired conclusions by $(2.21)$.

\section{Proof of Theorem 1.13}

Recall that the standard dyadic grid in $\mathbb{R}^{n}$ consists of all cubes of the form

$$
2^{-k}\left([0,1)^{n}+j\right), k \in \mathbb{Z}, j \in \mathbb{Z}^{n} .
$$

Denote the standard grid by $\mathcal{D}$.

As usual, by a general dyadic grid $\mathscr{D}$, we mean a collection of cube with the following properties: (i) for any cube $Q \in \mathscr{D}$, it side length $\ell(Q)$ is of the form $2^{k}$ for some $k \in \mathbb{Z}$; (ii) for any cubes $Q_{1}, Q_{2} \in \mathscr{D}, Q_{1} \cap Q_{2} \in\left\{Q_{1}, Q_{2}, \emptyset\right\}$; (iii) for each $k \in \mathbb{Z}$, the cubes of side length $2^{k}$ form a partition of $\mathbb{R}^{n}$.

The following lemma was established in [15].

Lemma 3.1. There exists $2^{n}$ dyadic grids $\mathscr{D}_{\alpha}$, such that for any cube $Q \subset \mathbb{R}^{n}$, there exists a cube $Q_{\alpha} \in \mathscr{D}_{\alpha}$ which satisfies that $Q \subset Q_{\alpha}$ and $\ell\left(Q_{\alpha}\right) \leq 6 \ell(Q)$.

For fixed $\alpha=1, \ldots, 2^{n}$, let $\mathcal{M}^{\mathscr{D} \alpha}$ be the maximal operator defined by

$$
\mathcal{M}^{\mathscr{D}_{\alpha}}\left(f_{1}, \ldots, f_{m}\right)(x)=\sup _{\substack{Q \ni x \\ Q \in \mathscr{D}_{\alpha}}} \prod_{j=1}^{m} \frac{1}{|Q|} \int_{Q}\left|f_{j}\left(y_{j}\right)\right| \mathrm{d} y_{j} .
$$

Similarly, for $i, l \in \mathbb{N}, 1 \leq i \leq m$, we define the maximal operator $\mathcal{M}_{i}^{l, \mathscr{D}_{\alpha}}$ by

$$
\mathcal{M}_{\Lambda}^{l, \mathscr{D}_{\alpha}}\left(f_{1}, \ldots, f_{m}\right)(x)=\sup _{\substack{Q \ni x \\ Q \in \mathscr{D} \alpha}}\left(\frac{1}{|Q|} \int_{Q}\left|f_{i}\left(y_{i}\right)\right| \mathrm{d} y_{i}\right) \prod_{\substack{1 \leq j \leq m, j \neq i}}\left(\frac{1}{\left|2^{l} Q\right|} \int_{2^{l} Q}\left|f_{i}\left(y_{j}\right)\right| \mathrm{d} y_{j}\right) .
$$


It then follows from Lemma 3.1 that

$$
\mathcal{M}\left(f_{1}, \ldots, f_{m}\right)(x) \lesssim \sum_{\alpha=1}^{2^{n}} \mathcal{M}^{\mathscr{D}_{\alpha}}\left(f_{1}, \ldots, f_{m}\right)(x)
$$

and

$$
\mathcal{M}_{i}^{l}\left(f_{1}, \ldots, f_{m}\right)(x) \lesssim \sum_{\alpha=1}^{2^{n}} \mathcal{M}_{i}^{l, \mathscr{D}_{\alpha}}\left(f_{1}, \ldots, f_{m}\right)(x)
$$

Associated with $\mathscr{D}_{\alpha}$, define the sharp maximal function $M^{\sharp, \mathscr{D}_{\alpha}}$ as

$$
M^{\sharp, \mathscr{D}_{\alpha}} f(x)=\sup _{\substack{Q \ni x \\ Q \in \mathscr{D}_{\alpha}}} \inf _{c \in \mathbb{C}} \frac{1}{|Q|} \int_{Q}|f(y)-c| \mathrm{d} y .
$$

As it was proved in $\left[21\right.$, p. 153], for $p \in(0, \infty)$ and $w \in A_{\infty}\left(\mathbb{R}^{n}\right)$,

$$
\|f\|_{L^{p}\left(\mathbb{R}^{n}, w\right)} \lesssim\left\|M^{\sharp, \mathscr{D}_{\alpha}} f\right\|_{L^{p}\left(\mathbb{R}^{n}, w\right)},
$$

provided that $\|M f\|_{L^{p}\left(\mathbb{R}^{n}, w\right)}<\infty$. Also, repeating the argument in [21, p. 153], we can verify that

$$
\|f\|_{L^{p, \infty}\left(\mathbb{R}^{n}, w\right)} \lesssim\left\|M^{\sharp, \mathscr{D}_{\alpha}} f\right\|_{L^{p, \infty}\left(\mathbb{R}^{n}, w\right)},
$$

provided that $\|M f\|_{L^{p, \infty}\left(\mathbb{R}^{n}, w\right)}<\infty$. Let $M_{\delta}^{\sharp, \mathscr{D}_{\alpha}} f(x)=\left[M^{\sharp, \mathscr{D}_{\alpha}}\left(|f|^{\delta}\right)(x)\right]^{1 / \delta}$ with $\delta \in(0, \infty)$.

The following lemma is a generalization of Lemma 8.1 in [4] in the setting of multi(sub)linear cases, and will play an important role in the proof of Theorem 1.13 .

Lemma 3.5. Let $q_{1}, \ldots q_{m} \in(1, \infty), q \in(1 / m, \infty)$ such that $1 / q=1 / q_{1}+\cdots+$ $1 / q_{m}$. Then for integer $1 \leq i \leq m, \delta \in(0,1 / m)$ and $\alpha=1, \ldots, 2^{n}$,

$(3.6) M_{\delta}^{\sharp, \mathscr{D}_{\alpha}}\left(\left\|\left\{\mathcal{M}^{\mathscr{D}_{\alpha}}\left(f_{1}^{k}, \ldots, f_{m}^{k}\right)\right\}\right\|_{l^{q}}\right)(x) \lesssim \mathcal{M}\left(\left\|\left\{f_{1}^{k}\right\}\right\|_{l^{q_{1}}}, \ldots,\left\|\left\{f_{m}^{k}\right\}\right\|_{l^{q_{m}}}\right)(x)$

and

$$
\begin{aligned}
M_{\delta}^{\sharp, \mathscr{D}_{\alpha}} & \left(\left\|\left\{\mathcal{M}_{i}^{l, \mathscr{D}_{\alpha}}\left(f_{1}^{k}, \ldots, f_{m}^{k}\right)\right\}\right\|_{l^{q}}\right)(x) \\
\lesssim & l M_{\delta}\left[\mathcal{M}_{i}^{l}\left(\left\|\left\{f_{1}^{k}\right\}\right\|_{l^{q_{1}}}, \ldots,\left\|\left\{f_{m}^{k}\right\}\right\|_{l^{q_{m}}}\right)\right](x) \\
& +\mathcal{M}\left(\left\|\left\{f_{1}^{k}\right\}\right\|_{l^{q_{1}}}, \ldots,\left\|\left\{f_{m}^{k}\right\}\right\|_{l^{q_{m}}}\right)(x) .
\end{aligned}
$$

Proof. We only prove (3.7). The proof of inequality (3.6) is similar and simpler, and will be omitted. For the sake of simplicity, we only prove (3.7) for $\mathscr{D}_{\alpha}=\mathcal{D}$. Let $x \in \mathbb{R}^{n}$ and $Q_{0}$ be a dyadic cube containing $x$. For each $y \in Q_{0}$ and integer $v$ with $1 \leq v \leq l$, let

$$
\mathrm{A}_{v}^{k}(y)=\left(\frac{1}{|Q|} \int_{Q}\left|f_{i}^{k}\left(y_{i}\right)\right| \mathrm{d} y_{i}\right) \prod_{\substack{1 \leq j \leq m \\ j \neq i}}\left(\frac{1}{\left|2^{l} Q\right|} \int_{2^{l} Q}\left|f_{j}^{k}\left(y_{j}\right)\right| \mathrm{d} y_{j}\right)
$$

with $Q$ the unique dyadic cube containing $y$ and $\ell(Q)=2^{-v} \ell\left(Q_{0}\right)$. Also, set

$$
\mathrm{A}_{l+1}^{k}(y)=\sup _{\substack{y \in Q \in \mathcal{D} \\ \ell(Q)<2^{-l} \ell\left(Q_{0}\right)}}\left(\frac{1}{|Q|} \int_{Q}\left|f_{i}^{k}\left(y_{i}\right)\right| \mathrm{d} y_{i}\right) \prod_{\substack{1 \leq j \leq m \\ j \neq i}}\left(\frac{1}{\left|2^{l} Q\right|} \int_{2^{l} Q}\left|f_{j}^{k}\left(y_{j}\right)\right| \mathrm{d} y_{j}\right) .
$$

Observe that for cubes $Q \subset Q_{0} \in \mathcal{D}$, if $\ell(Q)<2^{-v} \ell\left(Q_{0}\right)$ for some $v \in \mathbb{N}$, then $2^{v} Q \subset 2 Q_{0}$. Thus,

$$
\mathrm{A}_{l+1}^{k}(y) \lesssim \mathcal{M}_{i}^{l}\left(f_{1}^{k} \chi_{2 Q_{0}}, \ldots, f_{m}^{k} \chi_{2 Q_{0}}\right)(y)
$$


It is easy to verify that

$$
\mathcal{M}_{i}^{l, \mathcal{D}}\left(f_{1}^{k}, \ldots, f_{m}^{k}\right)(y)=\max \left\{\mathrm{A}_{1}^{k}(y), \ldots, \mathrm{A}_{l}^{k}(y), \mathrm{A}_{l+1}^{k}(y), \mathrm{D}_{l}^{k}\left(f_{1}^{k}, \ldots, f_{m}^{k}\right)\right\},
$$

with

$$
\mathrm{D}_{l}^{k}\left(f_{1}^{k}, \ldots, f_{m}^{k}\right)=\sup _{\substack{Q \in \mathcal{D} \\ Q_{0} \subset Q}}\left(\frac{1}{|Q|} \int_{Q}\left|f_{i}^{k}\left(y_{i}\right)\right| \mathrm{d} y_{i}\right) \prod_{\substack{1 \leq j \leq m \\ j \neq i}}\left(\frac{1}{\left|2^{l} Q\right|} \int_{2^{l} Q}\left|f_{j}^{k}\left(y_{j}\right)\right| \mathrm{d} y_{j}\right) .
$$

Let $C_{0}=\left\|\left\{\mathrm{D}_{l}^{k}\left(f_{1}^{k}, \ldots, f_{m}^{k}\right)\right\}\right\|_{l^{q}}$. Recall that $\delta<1 / m<q$. As in the proof of Lemma 2.15, we can write

$$
\begin{aligned}
& \|\|\left\{\mathcal{M}_{i}^{l, \mathcal{D}}\left(f_{1}^{k}, \ldots, f_{m}^{k}\right)(y)\right\} \|_{l^{q}}^{\delta}-\left|C_{0}\right|^{\delta} \mid \\
& \quad \leq\left\|\left\{\mathcal{M}_{i}^{l, \mathcal{D}}\left(f_{1}^{k}, \ldots, f_{m}^{k}\right)(y)-\mathrm{D}_{l}^{k}\left(f_{1}^{k}, \ldots, f_{m}^{k}\right)\right\}\right\|_{l^{q}}^{\delta} \\
& \quad \lesssim\left\|\left\{A_{1}^{k}(y)+\cdots+A_{l}^{k}(y)\right\}\right\|_{l^{q}}^{\delta}+\left\|\left\{\mathcal{M}_{i}^{l}\left(f_{1}^{k} \chi_{2 Q_{0}}, \ldots, f_{m}^{k} \chi_{2 Q_{0}}\right)(y)\right\}\right\|_{l^{q}}^{\delta} .
\end{aligned}
$$

We now estimate $\left\|\left\{A_{1}^{k}(y)+\cdots+A_{l}^{k}(y)\right\}\right\|_{l^{q}}$. For each $1 \leq v \leq l$ and $y \in Q_{0}$, applications of Hölder's inequality and Minkowski's inequality give us that

$$
\begin{aligned}
\left\|\left\{\mathrm{A}_{v}^{k}(y)\right\}\right\|_{l^{q}} \lesssim & \left(\frac{1}{|Q|} \int_{Q}\left\|\left\{f_{i}^{k}\left(y_{i}\right)\right\}\right\|_{l^{q_{i}}} \mathrm{~d} y_{i}\right) \\
& \times \prod_{\substack{1 \leq j \leq m \\
j \neq i}}\left(\frac{1}{\left|2^{l} Q\right|} \int_{2^{l} Q}\left\|\left\{f_{j}^{k}\left(y_{j}\right)\right\}\right\|_{l^{q_{j}}} \mathrm{~d} y_{j}\right) \\
& \lesssim \mathcal{M}_{i}^{l}\left(\left\|\left\{f_{1}^{k}\right\}\right\|_{l^{q_{1}}}, \ldots,\left\|\left\{f_{m}^{k}\right\}\right\|_{l^{q_{m}}}\right)(y)
\end{aligned}
$$

This, in turn, implies that

$$
\begin{aligned}
& \left(\frac{1}{\left|Q_{0}\right|} \int_{Q_{0}}\left\|\left\{A_{1}^{k}(y)+\cdots+A_{l}^{k}(y)\right\}\right\|_{l^{q}}^{\delta} \mathrm{d} y\right)^{1 / \delta} \\
& \quad \lesssim l M_{\delta}\left(\mathcal{M}_{i}^{l}\left(\left\|\left\{f_{1}^{k}\right\}\right\|_{l^{q_{1}}}, \ldots,\left\|\left\{f_{m}^{k}\right\}\right\|_{l^{q_{m}}}\right)(x) .\right.
\end{aligned}
$$

We can now conclude the proof of Lemma 3.5. Recall that

$$
\mathcal{M}_{\Lambda}^{l}\left(f_{1}^{k}, \ldots, f_{m}^{k}\right)(z) \lesssim \prod_{j=1}^{m} M f_{j}^{k}(z)
$$

It is obvious that is $\mathcal{M}_{i}^{l}$ is bounded from $L^{1}\left(l^{q_{1}} ; \mathbb{R}^{n}\right) \times \cdots \times L^{1}\left(l^{q_{m}} ; \mathbb{R}^{n}\right)$ to $L^{1 / m, \infty}\left(l^{q} ; \mathbb{R}^{n}\right)$ with bounded independent of $i$ and $l$. As in the proof of Kolmorgov's inequality, we deduce that

$$
\begin{aligned}
& \left(\frac{1}{\left|Q_{0}\right|} \int_{Q_{0}}\left\|\left\{\mathcal{M}_{i}^{l}\left(f_{1}^{k} \chi_{2 Q_{0}}, \ldots, f_{m}^{k} \chi_{2 Q_{0}}\right)(y)\right\}\right\|_{l^{q}}^{\delta} \mathrm{d} y\right)^{\frac{1}{\delta}} \\
& \quad \lesssim \prod_{j=1}^{m}\left(\frac{1}{\left|2 Q_{0}\right|} \int_{2 Q_{0}}\left\|\left\{f_{j}^{k}(z)\right\}\right\|_{l^{q_{j}}} \mathrm{~d} z\right) \\
& \quad \lesssim \mathcal{M}\left(\left\|\left\{f_{1}^{k}\right\}\right\|_{l^{q_{1}}}, \ldots,\left\|\left\{f_{m}^{k}\right\}\right\|_{l^{q_{m}}}\right)(x) .
\end{aligned}
$$

Combining the estimates (3.8) and (3.9) then leads to that

$$
\begin{aligned}
& \left(\left.\frac{1}{\left|Q_{0}\right|} \int_{Q_{0}}\left|\left\|\left\{\mathcal{M}_{i}^{l, \mathcal{D}}\left(f_{1}^{k}, \ldots, f_{m}^{k}\right)(y)\right\}\right\|_{l^{q}}^{\delta}-\right| C_{0}\right|^{\delta} \mid \mathrm{d} y\right)^{\frac{1}{\delta}} \\
& \quad \lesssim l M_{\delta}\left(\mathcal{M}_{i}^{l}\left(\left\|\left\{f_{1}^{k}\right\}\right\|_{l^{q_{1}}}, \ldots,\left\|\left\{f_{m}^{k}\right\}\right\|_{l^{q_{m}}}\right)(x)\right. \\
& \quad+\mathcal{M}\left(\left\|\left\{f_{1}^{k}\right\}\right\|_{l^{q_{1}}}, \ldots,\left\|\left\{f_{m}^{k}\right\}\right\|_{l^{q_{m}}}\right)(x),
\end{aligned}
$$


and leads to the desired conclusion for $\mathcal{M}_{i}^{l, \mathcal{D}}$.

Proof of Theorem 1.13. We only prove the conclusion for $\mathcal{M}_{i}(1 \leq i \leq m)$. Obviously, it suffices to consider the case that $\left\{f_{1}^{k}\right\}, \ldots,\left\{f_{m}^{k}\right\}$ are finite sequences.

We first consider the case $p_{1}, \ldots, p_{m} \in(1, \infty)$. Let $q_{1}, \ldots, q_{m} \in(1, \infty), \vec{w}=$ $\left(w_{1}, \ldots, w_{m}\right) \in A_{\vec{P}}\left(\mathbb{R}^{n m}\right), \alpha=1, \ldots, 2^{n}$ and $\delta \in(0,1 / m)$, we obtain from (3.3), Lemma 3.5 and Lemma 2.14, that

$$
\begin{aligned}
& \left\|\left\{\mathcal{M}_{i}^{l, \mathscr{D}_{\alpha}}\left(f_{1}^{k}, \ldots, f_{m}^{k}\right)\right\}\right\|_{L^{p}\left(l^{q} ; \mathbb{R}^{n}, \nu_{\vec{w}}\right)} \\
& \lesssim\left\|M_{\delta}^{\sharp, \mathscr{D}_{\alpha}}\left(\left\|\left\{\mathcal{M}_{i}^{l, d}\left(f_{1}^{k}, \ldots, f_{m}^{k}\right)\right\}\right\|_{l^{q}}\right)\right\|_{L^{p}\left(\mathbb{R}^{n}, \nu_{\vec{w}}\right)} \\
& \lesssim l\left\|\mathcal{M}_{i}^{l}\left(\left\|\left\{f_{1}^{k}\right\}\right\|_{l^{q_{1}}}, \ldots,\left\|\left\{f_{m}^{k}\right\}\right\|_{l^{q_{m}}}\right)\right\|_{L^{p}\left(\mathbb{R}^{n}, \nu_{\vec{w}}\right)} \\
& \quad+\left\|\mathcal{M}\left(\left\|\left\{f_{1}^{k}\right\}\right\|_{l^{q_{1}}}, \ldots,\left\|\left\{f_{m}^{k}\right\}\right\|_{l^{q_{m}}}\right)\right\|_{L^{p}\left(\mathbb{R}^{n}, \nu_{\vec{w}}\right)} \\
& \lesssim 2^{n l} 2^{-\theta l} l \prod_{j=1}^{m}\left\|\left\{f_{j}^{k}\right\}\right\|_{L^{p_{j}}\left(l^{q_{j}} ; \mathbb{R}^{n}, w_{j}\right)},
\end{aligned}
$$

since $\nu_{\vec{w}} \in A_{p / \delta}\left(\mathbb{R}^{n}\right)$. This, via (3.2), yields

$$
\left\|\left\{\mathcal{M}_{i}^{l}\left(f_{1}^{k}, \ldots, f_{m}^{k}\right)\right\}\right\|_{L^{p}\left(l^{q} ; \mathbb{R}^{n}, \nu_{\vec{w}}\right)} \lesssim l 2^{n l} 2^{-\theta l} \prod_{j=1}^{m}\left\|\left\{f_{j}^{k}\right\}\right\|_{L^{p_{j}\left(l^{q_{j}} ; \mathbb{R}^{n}, w_{j}\right)}} .
$$

Observe that for $q \in(1, \infty)$,

$$
\left\|\left\{\mathcal{M}_{i}\left(f_{1}^{k}, \ldots, f_{m}^{k}\right)(x)\right\}\right\|_{l^{q}} \leq \sum_{l=1}^{\infty} 2^{-l n}\left\|\left\{\mathcal{M}_{i}^{l}\left(f_{1}^{k}, \ldots, f_{m}^{k}\right)(x)\right\}\right\|_{l^{q}}
$$

and for $q \in(0,1]$,

$$
\left\|\left\{\mathcal{M}_{i}\left(f_{1}^{k}, \ldots, f_{m}^{k}\right)(x)\right\}\right\|_{l^{q}}^{q} \leq \sum_{l=1}^{\infty} 2^{-n l q}\left\|\left\{\mathcal{M}_{i}^{l}\left(f_{1}^{k}, \ldots, f_{m}^{k}\right)(x)\right\}\right\|_{l^{q}}^{q} .
$$

Therefore,

$$
\left\|\left\{\mathcal{M}_{i}\left(f_{1}^{k}, \ldots, f_{m}^{k}\right)\right\}\right\|_{L^{p}\left(l q ; \mathbb{R}^{n}, \nu_{\vec{w}}\right)} \leq \sum_{l=1}^{\infty} 2^{-n l}\left\|\left\{\mathcal{M}_{i}^{l}\left(f_{1}^{k}, \ldots, f_{m}^{k}\right)\right\}\right\|_{L^{p}\left(l q ; \mathbb{R}^{n}, \nu_{\vec{w}}\right)},
$$

when $p, q \in(1, \infty)$; and

$$
\left\|\left\{\mathcal{M}_{i}\left(f_{1}^{k}, \ldots, f_{m}^{k}\right)\right\}\right\|_{L^{p}\left(l^{q} ; \mathbb{R}^{n}, \nu_{\vec{w}}\right)}^{p} \leq \sum_{l=1}^{\infty} 2^{-n l p}\left\|\left\{\mathcal{M}_{i}^{l}\left(f_{1}^{k}, \ldots, f_{m}^{k}\right)\right\}\right\|_{L^{p}\left(l^{q} ; \mathbb{R}^{n}, \nu_{\vec{w}}\right)}^{p},
$$

when $q \in(1, \infty)$ and $p \in(0,1]$ or $q \in(0,1]$ and $p \in(0, q]$; and

$$
\left\|\left\{\mathcal{M}_{i}\left(f_{1}^{k}, \ldots, f_{m}^{k}\right)\right\}\right\|_{L^{p}\left(l^{q} ; \mathbb{R}^{n}, \nu_{\vec{w}}\right)}^{q} \leq \sum_{l=1}^{\infty} 2^{-n l q}\left\|\left\{\mathcal{M}_{i}^{l}\left(f_{1}^{k}, \ldots, f_{m}^{k}\right)\right\}\right\|_{L^{p}\left(l q ; \mathbb{R}^{n}, \nu_{\vec{w}}\right)}^{q},
$$

when $q \in(0,1]$ and $p \in(q, \infty)$. We now deduce from (3.10) that

$$
\left\|\left\{\mathcal{M}_{i}\left(f_{1}^{k}, \ldots, f_{m}^{k}\right)\right\}\right\|_{L^{p}\left(l^{q} ; \mathbb{R}^{n}, \nu_{\vec{w}}\right)} \lesssim \prod_{j=1}^{m}\left\|\left\{f_{j}^{k}\right\}\right\|_{L^{p_{j}\left(l^{q_{j}} ; \mathbb{R}^{n}, w_{j}\right)}} .
$$

We now consider the case that $\min _{1 \leq j \leq m} p_{j}=1$. For $q_{1}, \ldots, q_{m} \in(1, \infty)$, $\vec{w}=\left(w_{1}, \ldots, w_{m}\right) \in A_{\vec{P}}\left(\mathbb{R}^{n m}\right), \alpha=1, \ldots, 2^{n}$ and $\delta \in(0,1 / m)$, we get from (3.4), Lemma 3.5 and Lemma 2.14, that

$$
\left\|\left\{\mathcal{M}_{i}^{l, \mathscr{D}_{\alpha}}\left(f_{1}^{k}, \ldots, f_{m}^{k}\right)\right\}\right\|_{L^{p, \infty}\left(l^{q} ; \mathbb{R}^{n}, \nu_{\vec{w}}\right)} \lesssim 2^{n l} 2^{-\theta l} l \prod_{j=1}^{m}\left\|\left\{f_{j}^{k}\right\}\right\|_{L^{p_{j}\left(l^{q_{j}} ; \mathbb{R}^{n}, w_{j}\right)}},
$$


which, together with (3.2) gives us that

$$
\left\|\left\{\mathcal{M}_{i}^{l}\left(f_{1}^{k}, \ldots, f_{m}^{k}\right)\right\}\right\|_{L^{p, \infty}\left(l q ; \mathbb{R}^{n}, \nu_{\vec{w}}\right)} \lesssim 2^{n l} 2^{-\theta l} l \prod_{j=1}^{m}\left\|\left\{f_{j}^{k}\right\}\right\|_{L^{p_{j}\left(l^{q_{j}} ; \mathbb{R}^{n}, w_{j}\right)}} .
$$

On the other hand, as in the inequality (2.20), we get from (3.11), (3.12) and (3.13) that

$$
\begin{aligned}
& \nu_{\vec{w}}\left(\left\{x \in \mathbb{R}^{n}:\left\|\left\{\mathcal{M}_{i}\left(f_{1}^{k}, \ldots, f_{m}^{k}\right)(x)\right\}\right\|_{l^{q}}>C_{\theta} \lambda\right\}\right) \\
& \quad \lesssim \sum_{l=1}^{\infty} \nu_{\vec{w}}\left(\left\{x \in \mathbb{R}^{n}:\left\|\left\{\mathcal{M}_{i}^{l}\left(f_{1}^{k}, \ldots, f_{m}^{k}\right)(x)\right\}\right\|_{l^{q}}>2^{n l} 2^{-\theta l / 2} \lambda\right\}\right) \\
& \quad \lesssim \lambda^{-p} \prod_{j=1}^{m}\left\|\left\{f_{j}^{k}\right\}\right\|_{L^{p_{j}}\left(l^{\left.q_{j} ; \mathbb{R}^{n}, w_{j}\right)} .\right.}^{p}
\end{aligned}
$$

This completes the proof of Theorem 1.13 .

\section{ACKNOWLEDGEMENTS}

The research of the first author was supported by the NNSF of China under grant \#11371370. The research of the second author is supported by the European Union through T. Hytönen's ERC Starting Grant 'Analytic-probabilistic methods for borderline singular integrals'. He is a member of the Finnish Centre of Excellence in Analysis and Dynamics Research.

\section{REFERENCES}

[1] K. F. Anderson and R. T. John, Weighted inequality for vector-valued maximal functions and singular integrals, Studia Math. 69 (1980), 19-31.

[2] R. R. Coifman and Y. Meyer, On commutators of singular integrals and bilinear singular integrals, Trans. Amer. Math. Soc., 212 (1975), 315-331.

[3] R. R. Coifman and Y. Meyer, Au delà des opérateurs pseudo-différentiels, Astériaque 57, 1978. 1-185.

[4] D. Cruz-Uribe, SFO, J. Martell and C. Pérez, Sharp weighted estimates for classical operators, Adv. Math. 229 (2012), 408-441.

[5] X. Duong, R. Gong, L. Grafakos, J. Li and L. Yan, Maximal operator for multilinear singular integrals with non-smooth kernels. Indiana Univ. Math. J. 58 (2009), 2517-2542.

[6] X. Duong, L. Grafakos, L. Yan, Multilinear operators with non-smooth kernels and commutators of singular integrals. Trans. Amer. Math. Soc. 362 (2010), 2089-2113.

[7] C. Fefferman and E. M. Stein, Some maximal operators, Amer. J. Math. 93 (1971), 107115.

[8] J. Garcia-Cuerva and J. L. Rubio de Francia, Weighted Norm Inequalities and Related Topics, North-Holland, Amsterdam, 1985.

[9] L. Grafakos and N. Kalton, Multilinear Calderón-Zygmund operators on Hardy spaces, Collect. Math. 52 (2001) 169-179.

[10] L. Grafakos, L. Liu and D. Yang, Multilple-weighted norm inequalities for maximal singular integrals with non-smooth kernels, Proc. Royal Soc. Edinb. 141A (2011), 755-775.

[11] L. Grafakos, J. M. Martell, Extrapolation of weighted norm inequalities for multivariable operators and applications, J. Geom. Anal. 14 (2004) 19-46.

[12] L. Grafakos and R. Torres, Multilinear Calderón-Zygmund theory, Adv. Math. 165 (2002), 124-164.

[13] L. Grafakos and R. Torres, Maximal operator and weighted norm inequalities for multilinear singular integrals, Indiana Univ. Math. J. 51 (2002), 1261-1276.

[14] G. Hu and Y. Zhu, Weighted norm inequalities with general weights for the commutator of Calderón, Acta Math. Sinica, English Ser. 29 (2013), 505-514.

[15] T. Hytönen and C. Pérez, Sharp weighted bounds involving $A_{\infty}$, Anal. PDE. 6 (2013), $777-818$.

[16] A. Lerner, S. Ombrossi, C. Pérez, R. H. Torres and R. Trojillo-Gonzalez, New maximal functions and multiple weights for the multilinear Calderón-Zygmund theorey, Adv. Math. 220 (2009), 1222-1264. 
[17] K. Li, K. Moen and W. Sun, The sharp weighted bound for multilinear maximal functions and Calderón-Zygmund operators, J. Four. Anal. Appl. 20 (2014), 751-765.

[18] K. Li and W. Sun, Weak and strong type weighted estimates for multilinear CalderónZygmund operators, Adv. Math. 254 (2014), 736-771

[19] E. Sawyer, Norm inequalities relating singular integrals and the maximal function. Studia Math. 75 (1983), 253-263.

[20] E. M. Stein, Singular Integrals and the Differential Properties of Functions, Princeton Univ. Press, Princeton, NJ, 1970.

[21] E. M. Stein, Harmonic Analysis, Real Variable Methods, Orthogonality, and Oscillatory Integrals, Princeton Univ. Press, Princeton, NJ. 1993

(G. Hu) Department of Applied Mathematics, Zhengzhou Information Science and Technology Institute, Zhengzhou 450001, P. R. China

E-mail address: guoenxx@163.com

(K. Li) Department of Mathematics and Statistics, P.O.B. 68 (Gustaf Hällströmin Katu 2B), FI-00014 University of Helsinki, Finland

E-mail address: kangwei.li@helsinki.fi 